\title{
Incremental Computation with Names
}

\author{
Extended Version
}

\author{
Matthew A. Hammer ${ }^{1,2}$ Jana Dunfield ${ }^{3} \quad$ Kyle Headley ${ }^{1,2} \quad$ Nicholas Labich $^{2}$ \\ Jeffrey S. Foster ${ }^{2}$ Michael Hicks ${ }^{2}$ David Van Horn ${ }^{2}$ \\ ${ }^{1}$ University of Colorado \\ Boulder, USA \\ 2 University of Maryland \\ ${ }^{3}$ University of British Columbia \\ College Park, USA \\ Vancouver, Canada
}

\begin{abstract}
Over the past thirty years, there has been significant progress in developing general-purpose, language-based approaches to incremental computation, which aims to efficiently update the result of a computation when an input is changed. A key design challenge in such approaches is how to provide efficient incremental support for a broad range of programs. In this paper, we argue that first-class names are a critical linguistic feature for efficient incremental computation. Names identify computations to be reused across differing runs of a program, and making them first class gives programmers a high level of control over reuse. We demonstrate the benefits of names by presenting NOMINAL ADAPTON, an ML-like language for incremental computation with names. We describe how to use NOMINAL ADAPTON to efficiently incrementalize several standard programming patterns-including maps, folds, and unfoldsand show how to build efficient, incremental probabilistic trees and tries. Since Nominal ADAPTON's implementation is subtle, we formalize it as a core calculus and prove it is from-scratch consistent, meaning it always produces the same answer as simply re-running the computation. Finally, we demonstrate that NOMINAL ADAPTON can provide large speedups over both from-scratch computation and ADAPTON, a previous state-of-the-art incremental computation system.
\end{abstract}

Categories and Subject Descriptors $\quad$ D.3.1 [Programming Languages]: Formal Definitions and Theory; D.3.3 [Programming Languages]: Language Constructs and Features; F.3.2 [Logics and Meanings of Programs]: Semantics of Programming Languages

Permission to make digital or hard copies of all or part of this work for personal or classroom use is granted without fee provided that copies are not made or distributed for profit or commercial advantage and that copies bear this notice and the full citation on the first page. Copyrights for components of this work owned by others than the author(s) must be honored. Abstracting with credit is permitted. To copy otherwise, or republish, to post on servers or to redistribute to lists, requires prior specific permission and/or a fee. Request permissions from permissions@ acm.org.

OOPSLA '15, October 25-30, 2015, Pittsburgh, PA, USA

Copyright is held by the owner/author(s). Publication rights licensed to ACM. ACM 978-1-4503-3689-5/15/10_..\$15.00.

http://dx.doi.org/10.1145/2814270.2814305
Keywords laziness, thunks, call-by-push-value (CBPV), demanded computation graph (DCG), incremental computation, self-adjusting computation, memoization, nominal matching, structural matching

\section{Introduction}

Memoization is a widely used technique to speed up running time by caching and reusing prior results (Michie 1968). The idea is simple - the first time we call a pure function $f$ on immutable inputs $\vec{x}$, we store the result $r$ in a memo table mapping $\vec{x}$ to $r$. Then on subsequent calls $f(\vec{y})$, we can return $r$ immediately if $\vec{x}$ and $\vec{y}$ match. Incremental computation (IC) (Pugh 1988) takes this idea a step further, aiming to reuse prior computations even if there is a small change in the input. Recent forms of IC as exemplified by self-adjusting computation (SAC) Acar 2005) and ADAPTON (Hammer et al.|2014) support mutable inputs, meaning that two calls to $f(\vec{x})$ might produce different results because values reachable from the same arguments $\vec{x}$ have been mutated. As such, before reusing a memoized result $r$, any inconsistencies are repaired via a process called change propagation.

An important goal of an IC system is to minimize the work performed in support of change propagation, and thus improve overall performance. Matching - the task of determining whether a call's arguments are "the same" as those of a memoized call - turns out to play a key role, as we show in this paper. The most common mechanism is structural matching, which traverses an input's structure to check whether each of its components match. To make it fast, implementations use variants of hash-consing (e.g., see Filliâtre and Conchon (2006) ) which, in essence, associates with a pointer a hash of its contents and compares pointers by hash.

Structural matching works well when memoizing pure computations over immutable data because such computations always produce the same result. But for IC involving mutable references, structural matching can be too specific and therefore too fragile. For example, suppose we map a function $f$ over a mutable list input $=[0,1,3]$ producing output $=\operatorname{map} f$ input $=[f 0, f 1, f 3]$. Next, suppose we mutate input by inserting 2 , so input becomes $[0,1,2,3]$. Finally, suppose we recompute map $f$ input, now $[f 0, f 1, \mathbf{f} 2, f 3]$, at- 
tempting to reuse as much of the prior computation as possible. Structural matching will successfully identify and reuse the recursive sub-call map $f$ [3], reusing the result [f 3]. More generally, it will reuse the mapped suffix after the inserted element, since the computation of this output is independent of the mutated prefix. However, structural matching will not match the sub-computations that map 0 to $f 0$ and 1 to $f 1$ because these sub-computations' outputs transitively include the newly inserted value of $f 2$ (via their tail pointers). As a result, an IC system that uses structural matching will rerun those sub-computations from scratch, recomputing $f 0$ and $f 1$ and allocating new list cells to hold the results. (Section 2 covers this example in detail.)

The key takeaway is that structural matching is too conservative-it was designed for immutable inputs, in which case a structural match produces a correct memoized result. But with IC using mutable inputs, a match need not return a correct result; rather, our aim should be to return a result that requires only a little work to repair. For our example, an ideal IC system would be able to memoize the prefix, repairing it by mutating the old output cell containing $f 1$ to insert $f 2$.

In this paper, we propose to overcome the deficiencies of structural matching for IC by employing an alternative matching strategy that involves names. We implement our solution in NOMINAL ADAPTON, an extension to the ADAPTON IC framework. Our new nominal matching strategy permits the programmer to explicitly associate a name-a firstclass (but abstract) value-with a pointer such that pointers match when their names are equal. A program produces names by generating them from existing names or other seed values. Returning to our example, we can add names to list cells such that an output cell's name is derived from the corresponding input cell's name. With this change, insertion into a list does not affect output cells' names, and hence we can successfully reuse the computation of map on the prefix before the inserted element. The particular naming strategy is explained in detail in Section 2, which also gives an overview of the NOMINAL ADAPTON programming model and shows how it improves performance on the map example compared to prior structural approaches.

Nominal matching is strictly more powerful than structural matching: The programmer can choose names however they wish, including mimicking structural matching by using hashing. That said, there is a risk that names could be used ambiguously, associating the same name with distinct pointers. In NOMINAL ADAPTON, if the programmer makes a mistake and uses a name ambiguously, efficient runtime checks detect this misuse and raise an exception. The use-once restriction can be limiting, however, so in addition to supporting first-class names, NOMINAL ADAPTON provides first-class namespaces-the same name can be reused as long as each use is in a separate namespace. For example, we can safely map different functions over the same list by wrapping the computations in separate namespaces. Section 3 illustrates several use cases of the NOMINAL ADAPTON programming model, presenting naming design patterns for incremental lists and trees and common computation patterns over them. We also propose a fundamental data structure for probabilistically balanced trees that works in a variety of applications.

We have formalized NOMINAL ADAPTON in a core calculus $\lambda_{\text {NomA }}$ and proved its incremental recomputation is from-scratch consistent, meaning it produces the same answer as would a recomputation from scratch. As such, mistakes from the programmer will never produce incorrect results. Section 4 presents our formalism and theorem.

We have implemented NOMINAL ADAPTON in OCaml as an extension to ADAPTON (Section 5). We evaluated our implementation by comparing it to ADAPTON on a set of subject programs commonly evaluated in the IC literature, including map, filter, reduce, reverse, median, mergesort, and quickhull. As a more involved example, we implemented an interpreter for an imperative programming language (IMP with arrays), showing that interpreted programs enjoy incrementality by virtue of using NOMINAL ADAPTON as the meta-language. Across our benchmarks, we find that ADAPTON is nearly always slower than NOMINAL ADAPTON (sometimes orders of magnitude slower), and is sometimes orders of magnitude slower than from-scratch computation. By contrast, NOMINAL ADAPTON uniformly enjoys speedups over from-scratch computation (up to $10900 \times$ ) as well as classic ADAPTON (up to $21000 \times$ ). (Section 6 describes our experiments.)

The idea of names has come up in prior incremental computation systems, but only in an informal way. For example, Acar and Ley-Wild (2009) includes a paragraph describing the idea of named references (there called "keys") in the DeltaML implementation of SAC. To our knowledge, our work is the first to formalize a notion of named computations in IC and prove their usage correct. We are also the first to empirically evaluate the costs and benefits of programmernamed references and thunks. Finally, the notion of firstclass namespaces, with the same determinization benefits as named thunks and references, is also new. (Section 7 discusses SAC and other related work in more detail.)

\section{Overview}

In this section we present NOMINAL ADAPTON and its programming model, illustrating how names can be used to improve opportunities for reuse. We start by introducing ADAPTON's approach to incremental computation, highlighting how NOMINAL ADAPTON extends its programming model with support for names. Next we use an example, mapping over a list, to show how names can be used to improve incremental performance. 


\subsection{Adapton and Nominal Adapton}

ADAPTON aims to reuse prior computations as much as possible after a change to the input. ADAPTON achieves this by memoizing a function call's arguments and results, reusing memoized results when the arguments match (via structural matching). In this section, we write memo(e) to indicate that the programmer wishes $e$ to be memoized 1

ADAPTON provides mutable references: ref $e$ allocates a memory location $p$ which it initializes to the result of evaluating $e$, and ! $p$ retrieves the contents of that cell. Changes to inputs are expressed via reference cell mutations; ADAPTON propagates the effect of such changes to update previous results. Like many approaches to incremental computation, ADAPTON distinguishes two layers of computation. Computations in the inner layer are incremental, but can only read and allocate references, while computations in the outer layer can change reference values (necessitating change propagation for the affected inner-layer computations) but are not themselves incremental. This works by having the initial incremental run produce a demanded computation graph (DCG), which stores values of memoized computations and tracks dependencies between those computations and references. Changes to mutable state "dirty" this graph, and change propagation "cleans" it, making its results consistent.

\subsection{Incremental Computation in ADAPTON}

As a running example, consider incrementalizing a program that maps over a list's elements. To support this, we define a list data structure that allows the tail to be imperatively modified by the outer context:

$$
\begin{aligned}
& \text { type 'a list }=\mathrm{Nil} \mid \text { Cons of 'a } * \text { ('a list) ref } \\
& \text { let rec map } \mathrm{f} x \mathrm{~s}=\operatorname{memo}(\text { match xs with } \\
& \mid \mathrm{Nil} \rightarrow \mathrm{Nil} \\
& \mid \text { Cons }(\mathrm{x}, \mathrm{xs}) \rightarrow \text { Cons }(\mathrm{f} x \text {, ref }(\text { map } \mathrm{f} ! \mathrm{xs})))
\end{aligned}
$$

This is a standard map function, except for two twists: the function body is memoized via memo, and the input and output Cons tails are reference cells. The use of memo here records function calls to map, identifying prior calls using the function $f$ and input list xs. In turn, $x$ s is either Nil or is identified by a value of type 'a and a reference cell. Hence, reusing the identity of references is critical to reusing calls to map via memo. Now we can create a list (in the outer layer) and map over it (in the inner layer):

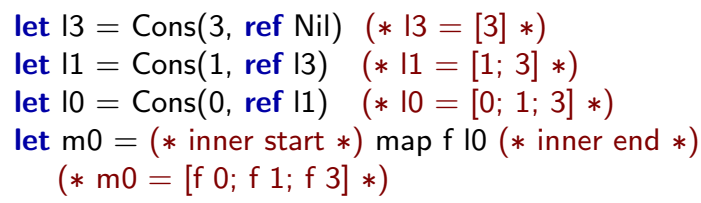

\footnotetext{
${ }^{1}$ Programmers actually have more flexibility thanks to ADAPTON's support for laziness, but laziness is orthogonal to names, which we focus on in this section. We discuss laziness in Section 4
}

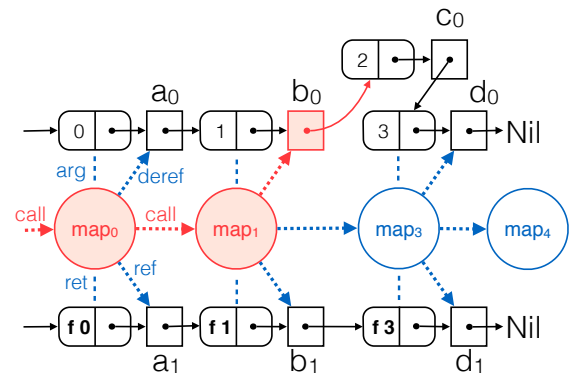

(a) ADAPTON, after insertion update

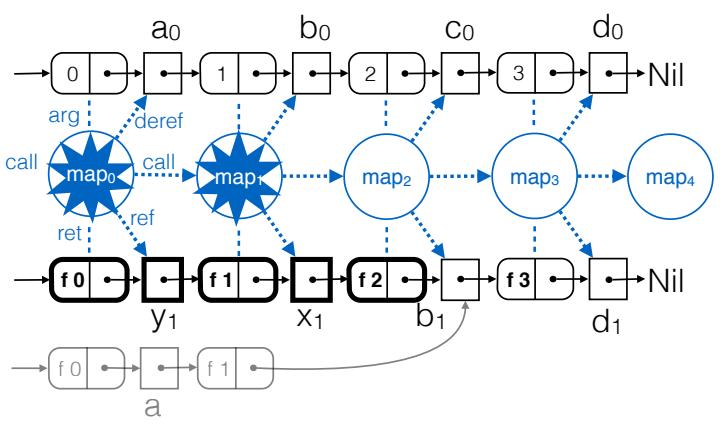

(b) ADAPTON, after change propagation

Figure 1: Incremental computation of map in ADAPTON

Suppose we change the input to map by inserting an element:

$$
(\mathrm{t} \mid 1):=\operatorname{Cons}(2, \operatorname{ref} \mid 3) \quad(* 10=[0 ; 1 ; 2 ; 3] *)
$$

Here, $\mathrm{tl}$ returns the tail of its list argument. After this change, $\mathrm{m} 0$ will be updated to [ $\mathrm{f} 0 ; \mathrm{f} 1 ; \mathrm{f} 2 ; \mathrm{f} 3]$. In the best case, computing $m 0$ should only require applying $f 2$ and inserting the result into the original output. However, ADAPTON performs much more work for the above code. Specifically, ADAPTON will recompute $f 0$ and $f 1$; if the change were in the middle of a longer list, it would recompute the entire prefix of the list before the change. In contrast, NOMINAL ADAPTON will only redo the minimal amount of work.

To understand why, consider Figure 1a, which illustrates what happens after the list update. In this figure, the initial input and output lists are shown in black at the top and the bottom of the figure, respectively. The middle of the figure shows the demanded computation graph (DCG), which records each recursive call of map and its dynamic dependencies. Here, nodes mapo, $\operatorname{map}_{1}, \operatorname{map}_{3}$ and $\operatorname{map}_{4}$ correspond to the four calls to map. For each call, the DCG records the arguments, the result, and the computation's effects. Here, the effects are: dereferencing a pointer; making a recursive call; and allocating a ref cell in the output list. We label the arrows/lines of the first node only, to avoid clutter; the same pattern holds for map ${ }_{1}$ and map $_{3}$.

In the input and output, the tail of each Cons cell (a rounded box) consists of a reference (a square box). The 
input list is labeled $a_{0}, b_{0}, d_{0}$, and the output list is labeled $a_{1}, b_{1}, d_{1}$. In ADAPTON, structural matching determines the labels chosen by the inner layer, and in particular, whenever the inner layer allocates a reference cell to hold a value that is already contained in an existing reference cell, it reuses this first cell and its label.

After the list is updated, ADAPTON dirties all the computations that transitively depend on the changed reference cell, $b_{0}$. The dirtied elements are shaded in red. Dirtying is how ADAPTON knows that previously memoized results cannot be reused safely without further processing. ADAPTON processes dirty parts of the DCG into clean computations on demand, when they are demanded by the outer program. To do so, it either re-executes the dirty computations, or verifies that they are unaffected by the original set of changes.

Figure $1 \mathrm{~b}$ shows the result of recomputing the output following the insertion change, using this mechanism. When the outer program recomputes map $f 10$ (shown as mapo), ADAPTON will clean (either recompute or reuse) the dirty nodes of the DCG. First, it re-executes computation $\operatorname{map}_{1}$, because it is the first dirty computation to be affected by the changed reference cell. We indicate reexecuted computations with stars in their DCG nodes. Upon re-execution, map $_{1}$ dereferences $b_{0}$ and calls map on the inserted Cons cell holding 2. This new call $\operatorname{map}_{2}$ calls $f$ 2 (not shown), dereferences $c_{0}$, calls map $_{3}$ 's computation map $f\left(\operatorname{Cons}\left(3, d_{0}\right)\right)$, allocates $b_{1}$ to hold its result and returns Cons $\left(f 3, d_{1}\right)$.

The recomputation of map $_{2}$ exploits two instances of reuse. First, when it calls map $f\left(\operatorname{Cons}\left(3, d_{0}\right)\right)$, ADAPTON reuses this portion of the DCG and the result it computed in the first run. ADAPTON knows that the prior result of map $f\left(\operatorname{Cons}\left(3, d_{0}\right)\right)$ is unchanged because map $_{3}$ is not dirty. Notice that even if the tail of the list were much longer, the prior computation of $\mathrm{map}_{3}$ could still be reused, since the insertion does not affect it.

Second, when $\operatorname{map}_{2}$ allocates the reference cell to hold the result of map $f\left(\operatorname{Cons}\left(3, d_{0}\right)\right.$, i.e. Cons $\left(f 3, d_{1}\right)$, it reuses and shares the existing reference cell $b_{1}$ that already holds this content. Notice that this maintains our labeling invariant, so we can continue to perform structural matching by comparing labels. As a side effect, it also improves performance by avoiding allocation of an (isomorphic) copy of the output ${ }^{2}$

\footnotetext{
${ }^{2}$ Note that the outer layer may cause two cells with the same contents to be labeled differently: If two cells are initially allocated with different contents, but then the outer layer mutates one cell to contain the contents of the other, the cells will still have different labels and hence will not match in ADAPTON. This is a practical implementation choice, since otherwise ADAPTON might need to do complicated heap operations to merge the identities of two cells. At worst, this choice causes ADAPTON to miss some minor opportunities for reuse. However, this choice is consistent with the standard implementation of hash-consing, which only aims to share immutable structures (Allen|1978. Filliâtre and Conchon|2006).
}

So far, ADAPTON has successfully reused subcomputations, but consider what happens next. When the call map 2 completes, $\operatorname{map}_{1}$ resumes control and allocates a reference cell to hold Cons $\left(f 2, b_{1}\right)$. Since no reference exists with this content, it allocates a fresh reference $x_{1}$. New references are shown in bold. The computation then returns Cons( $f 1$, $\left.x_{1}\right)$, which does not match its prior return value Cons $(f 1$, $\left.b_{1}\right)$. Since this return value has changed from the prior run, ADAPTON re-runs map 1 's caller, mapo. This consists of rerunning $f 0$, reusing the (just recomputed, hence no longer dirty) computation $\operatorname{map}_{1}$, and allocating a reference to hold its result Cons $\left(f 1, x_{1}\right)$. Again, no reference exists yet with this content ( $x_{1}$ is a fresh tail pointer), so ADAPTON allocates a fresh cell $y_{1}$. Finally, the call to mapo completes, returning a new list prefix with the same content ( $\mathrm{f}$ and $f$ 1 ) as in the first run, but with new reference cell identities $\left(\mathrm{y}_{1}\right.$ and $\left.\mathrm{x}_{1}\right)$.

In this example, small changes cascade into larger changes because ADAPTON identifies reference cells structurally based on their contents. Thus, the entire prefix of the output list before the insertion is reallocated and recomputed, which is much more work than should be necessary.

\subsection{The Nominal Approach}

We can solve the problems with structural matching by giving the programmer explicit names to control reuse. In this particular case, we aim to avoid re-computing map mo $_{0}$ and any preceding computations. In particular, we wish to recompute only map $_{1}$ (since it reads a changed pointer) and to compute $\operatorname{map}_{2}$, the mapping for the inserted Cons cell.

The first step is to augment mutable lists with names provided by NOMINAL ADAPTON:

$$
\text { type list }=\mathrm{Nil} \mid \text { Cons of int } * \text { name } * \text { (list ref) }
$$

Globally fresh names are generated either non-deterministically via new, or from an existing name via fork. In particular, fork $\mathrm{n}$ returns a pair of distinct names based on the name $\mathrm{n}$ with the property that it always returns the same pair of names given the same name $\mathrm{n}$. In this way, the inner layer can deterministically generate additional names from a given one to enable better reuse. Finally, when the programmer allocates a reference cell, she explicitly indicates which name to use, e.g. $\operatorname{ref}(n, 1)$ instead of $\operatorname{ref}(1)$.

Now, when the list is created, the outer layer calls new to generate fresh, globally distinct names for each Cons cell:

$$
\begin{array}{ll}
\text { let } I 3=\operatorname{Cons}(3, \text { new, } \operatorname{ref}(\text { new, Nil })) & (* I 3=[3] *) \\
\text { let } I 1=\operatorname{Cons}(1, \text { new, } \operatorname{ref}(\text { new, I3 })) & (* I 1=[1 ; 3] *) \\
\text { let } I 0=\operatorname{Cons}(0, \text { new, } \operatorname{ref}(\text { new, I1 })) & (* I 0=[0 ; 1 ; 3] *)
\end{array}
$$

When the inner layer computes with the list, it uses the names in each Cons cell to indicate dependencies between the inputs and outputs of the computation. In particular, we rewrite map as follows: 


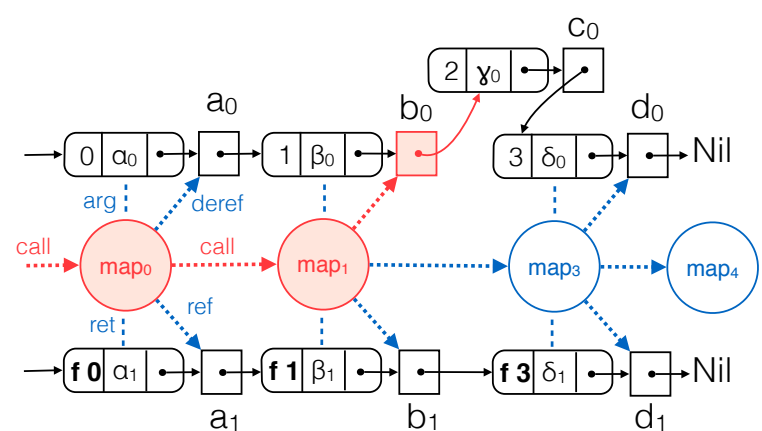

(a) NOMinal AdAPTON, after insertion update

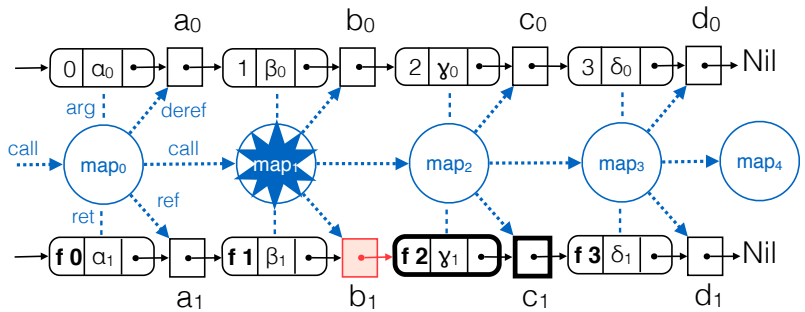

(b) Nominal Adapton, after change propagation

Figure 2: IC of map with NOMINAL ADAPTON

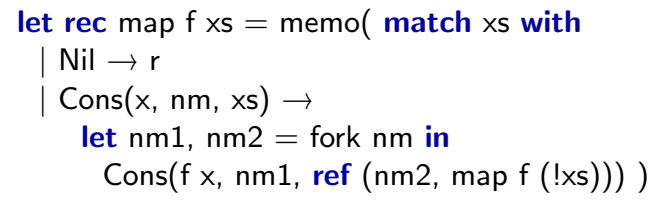

Unlike the outer program, which chooses reference names using new, the inner program uses fork to relate the names and references in the output list to the names in the input list.

Now consider applying this function and making the same change as above:

let $m 0=\operatorname{map} f 10$ in

$(t \mid l 1):=\operatorname{Cons}(2$, new, ref $\mid 3)$

Figure 2 shows what happens. The initial picture in Figure 2a is similar to the structural case in Figure 1a, except the input and output lists additionally contain names $\alpha_{0}, \beta_{0}, \gamma_{0}$ and $\delta_{0}$. The first part of the recomputation is the same: NOMINAL ADAPTON recomputes map ${ }_{1}$, which reads the mutated reference $b_{0}$. In turn, it recomputes $\operatorname{map}_{2}$, which reuses the call to $\operatorname{map}_{3}$ to compute Cons(f $\left.2, \gamma_{1}, c_{1}\right)$. The recomputation of $\operatorname{map}_{2}$ returns a different value than in the prior run, with the new head value $f 2$.

At this point, the critical difference occurs. Even though the result of map $_{2}$ is distinct from any list in the prior run, the call map ${ }_{1}$ allocates the same ref cell $b_{0}$ as before, because the name it uses for this allocation, $\beta_{1}$, is the same as before. In the figure, fork $\beta_{0} \mapsto\left(\beta_{1}, \beta_{2}\right)$, where $\beta_{1}$ becomes the name in the output list and $b_{1}=$ ref $\beta_{2}$ identifies the reference cell in its tail. NOMINAL ADAPTON dirties the reference $b_{1}$, to ensure that any dependent computations will be cleaned before their results are reused. Due to this reuse, the result of the call map $_{1}$ is identical to its prior result: The value of $f 1$ is unaffected, and the tail pointer $b_{1}$ was reused exactly. Next, NOMINAL ADAPTON examines mapo and all prior calls. Because the return value of map $_{1}$ did not change, NOMINAL ADAPTON simply marks the DCG node for its caller, mapo, as clean; no more re-evaluation occurs. This cleaning step breaks the cascade of changes that occurred under ADAPTON. Prior computations are now clean, because they only depend on clean nodes.

As a result of this difference in behavior, NOMINAL ADAPTON is able to reuse all but two calls to function $f$ for an insertion at any index $i$, while ADAPTON will generally re-execute all $i-1$ calls to function $f$ that precede the inserted cell. Moreover, ADAPTON allocates a new copy of the output prefix (from 0 to $i$ ), while NOMINAL ADAPTON reuses all prior allocations. Our experiments (Section 6) confirm that these differences make ADAPTON over $10 \times$ slower than NOMINAL ADAPTON, even for medium-sized lists (10k elements) and cheap instances of $f$ (integer arithmetic).

\subsection{Enforcing that Nominal Matching is Correct}

Putting the task of naming in the programmer's hands can significantly improve performance, but opens the possibility of mistakes that lead to correctness problems. In particular, a programmer could use the same name for two different objects:

$$
\begin{aligned}
& \text { let } y=\text { ref } n 1 \\
& \text { let } z=\text { ref } n \text { false }
\end{aligned}
$$

Double use leads to problems since the variables $y$ and $z$ have distinct types, yet they actually reference the same nominal object, the number or boolean named $\mathrm{n}$. Consequently, the dereferenced values of $y$ and $z$ are sensitive to the order of the allocations above, where the last allocation "wins." This imperative behavior is undesirable because it is inconsistent with our desired from-scratch semantics, where allocation always constructs new objects.

To forbid double use errors, our implementation uses an efficient dynamic check. As the DCG evolves during program execution, NOMINAL ADAPTON maintains a stack of DCG nodes, its force stack, which consists of those DCG nodes currently being forced (evaluated, re-evaluated, or reused). When nominal matching re-associates a name with a different value or computation than a prior usage, it overwrites information in the DCG, and it dirties the old use and its transitive dependencies in the DCG. To check that a nominal match is unambiguous, we exploit a key invariant: A name is used ambiguously by a nominal match if and only if one or more DCG nodes on the force stack are dirtied when said nominal match occurs. If no such node exists, then the name is unambiguous. NOMINAL ADAPTON implements this check by maintaining, for each DCG node, a bit that is set and unset when the node is pushed and popped 
from the force stack, respectively. This implementation is very efficient, with $\mathrm{O}(1)$ overhead.

Returning to the example above, the allocation of $z$ nominally matches the allocation on the prior line, for $y$. Since 1 and false are not equal, the nominal match dirties the DCG node that allocates 1 , which is also the "current" DCG node, and thus is on the top of the force stack. Hence, NOMINAL ADAPTON raises an exception at the allocation of $z$, indicating that $\mathrm{n}$ is used ambiguously.

Note that because this check is dynamic and based on the DCG, it works even when ambiguous name uses are separated across function or module boundaries. This is important since, in our experience, most name reuse errors are not nearly as localized as the example above. This dynamic check was essential for our own development process; without it, nominal mistakes were easy to make and nearly impossible to diagnose.

\subsection{Namespaces}

Unfortunately, forbidding multiple uses of names altogether prevents many reasonable coding patterns. For example, suppose we want to map an input list twice:

$$
\begin{aligned}
& \text { let } \mathrm{ys}=\text { map } \mathrm{f} \text { input_list } \\
& \text { let } \mathrm{zs}=\text { map } \mathrm{g} \text { input_list }
\end{aligned}
$$

Recall that in the Cons case of map we use each name in the input list to create a corresponding name in the output list. As such, the two calls to map result in ys and zs having cells with the same names, which is forbidden by our dynamic check.

We can address this problem by creating distinct namespaces for the distinct functions ( $f$ versus $g$ ), where the same names in two different namespaces are treated as distinct. A modified version of map using namespaces would be written thus:

$$
\text { let map' } \mathrm{n} \text { h xs }=\operatorname{nest}(\mathrm{ns}(\mathrm{n}) \text {, map } \mathrm{h} \times \mathrm{s})
$$

The code nest(s,e) performs the nested computation e in namespace $s$, and the code $n s(n)$ creates a namespace from a given name $n$. Just as with references, we must be careful about how namespaces correspond across different incremental runs, and thus we seed a namespace with a given name. Now, distinct callers can safely call map' with distinct names:

$$
\begin{aligned}
& \text { let } \mathrm{n} 1, \mathrm{n} 2=(\text { new, new }) \text { in } \\
& \text { let } \mathrm{xs}=\text { map }^{\prime} \mathrm{n} 1 \mathrm{f} \text { input_list in } \\
& \text { let } \mathrm{ys}=\text { map }^{\prime} \mathrm{n} 2 \mathrm{~g} \text { input_list in }
\end{aligned}
$$

The result is that each name in the input list is used only once per namespace: Names in map $f$ will be associated with the first namespace (named by $\mathrm{n} 1$ ), and names in map $\mathrm{g}$ will associate with the second namespace (named by n2).

Section summary. The use of names allows the programmer to control (1) how mutable reference names are chosen the first time, and (2) how to selectively reuse and overwrite these references to account for incremental input changes from the outer layer. These names are transferred from input to output through the data structures that they help identify (the input and output lists here), by the programs that process them (such as map). Sometimes we want to use the same name more than once, in different program contexts (e.g., map $f$. versus map $g$ ·); we distinguish these program contexts using namespaces.

\section{Programming with Names}

While NOMINAL ADAPTON's names are a powerful tool for improving incremental reuse, they create more work for the programmer. In our experience so far, effective name reuse follows easy-to-understand patterns. Section 3.1 shows how to augment standard data structures-lists and trees-and operations over them-maps, folds, and unfolds - to incorporate names in a way that supports effective reuse. Section 3.2 describes probabilistic tries, a nominal data structure we developed that efficiently implements incremental maps and sets. Finally, Section 3.3 describes our implementation of an incremental IMP interpreter that takes advantage of these data structures to support incremental evaluation of its imperative input programs. The benefits of these patterns and structures are measured precisely in Section 6

\subsection{General Programming Patterns}

Practical functional programs use a wide variety of programming patterns; three particularly popular ones are mapping, folding, and unfolding. We consider them here in the context of lists and trees.

Mapping. Maps traverse a list (as in Section 2) or tree and produce an output structure that has a one-to-one correspondence with the input structure. We have already seen how to incrementalize mapping by associating a name with each element of the input list and using fork to derive a corresponding name for each element in the output list, thereby avoiding spurious recomputation of whole list prefixes on a change.

Folding. Folds traverse a list or tree and reduce subcomputations to provide a final result. Examples are summing list elements or finding the minimum element in a tree.

If we implement folding in a straightforward way in NOMINAL ADAPTON, the resulting program tends to perform poorly. The problem is that every step in a list-based reduction uses an accumulator or result that induces a global dependency on all prior steps-i.e., every step depends on the entire prefix or the entire suffix of the sequence, meaning that any change therein necessitates recomputing the step.

The solution is to use an approach from parallel programming: Use trees to structure the input data, rather than lists, to permit expressing independence between sub-problems. Consider the following code, which defines a type tree for trees of integers: 
type tree $=$ Leaf $\mid$ Bin of name $*$ int $*($ tree ref $) *($ tree ref)

Like lists, these trees use refs to permit their recursive structure to change incrementally, and each tree node includes a name. We can reduce over this tree in standard functional style:

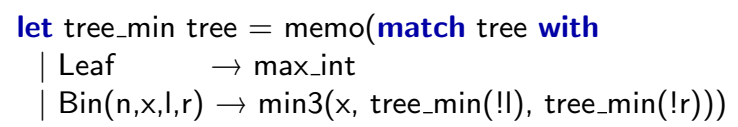

If we later update the tree and recompute, we can reuse subtree minimum computations, because the names are stable in the tree.

Below, we show the original input tree alongside two illustrations (also depicted as trees) of which element of each subtree is the minimum element, before and after the replacement of element 1 with the new element 9:

\begin{tabular}{|c|c|c|}
\hline Original tree & $\begin{array}{l}\text { Minimums } \\
\text { (pre-change) }\end{array}$ & $\begin{array}{c}\text { Minimums } \\
\text { (post-change) }\end{array}$ \\
\hline$V^{14} \cdot v^{6}$ & $V^{4} \cdot v^{6}$ & $94 \cdot 6$ \\
\hline
\end{tabular}

Notice that while the first element 1 changed to 9 , this only affects the minimum result along one path in the tree: the path from the root to the changed element. In contrast, if we folded the sequence naively as a list, all the intermediate computations of the minimum could be affected by a change to the first element (or last element, depending on the fold direction). By contrast, the balanced tree structure (with expected logarithmic depth) overcomes this problem by better isolating independent subcomputations.

Pleasingly, as first shown by Pugh and Teitelbaum(1989), we can efficiently build a tree probabilistically from an input list, and thus transfer the benefits of incremental tree reuse to list-style computations. Building such a tree is an example of unfolding, described next.

Unfolding. Unfolds iteratively generate lists or trees using a "step function" with internal state. As just mentioned, one example is building a balanced tree from a list. Unfortunately, if we implement unfolding in a straightforward way, incremental computation suffers. In particular, we want similar lists (related by small edits) to lead to similar trees, with many common subtrees; meanwhile, textbook algorithms for building balanced trees, such as AVL trees and splay trees, are too sensitive to changes to individual list elements.

The solution to this problem is to construct a probabilistically balanced tree, with expected $\mathrm{O}(\log n)$ height for input list $n$. The height of each element in the resulting tree is determined by a function that counts the number of trailing zero bits in a hash of the given integer. For example, given input elements $[a, b, c, d, e, f]$ with heights $[0,1,0,2,1,0]$, re- spectively, then our tree-construction function will produce the binary tree shown below:



Pugh and Teitelbaum (1989) showed that this procedure induces a probabilistically balanced tree, with similar lists inducing similar trees, as desired. Further, each distinct list of elements maps to exactly one tree structure. This property is useful in NOMINAL ADAPTON, since a canonical structure is more likely to be reused than one that can exhibit more structural variation. While past work has considered incremental computations over such balanced trees, in this work we find that the construction of the tree from a mutable, changing sequence can also be efficiently incrementalized (Pugh's work focused only on a pure outer program).

\subsection{Probabilistic Tries}

Inspired by probabilistic trees, we developed efficient, incremental probabilistic tries, which use a different naming pattern in which certain names are external to the data structure.

We define tries as binary trees whose nodes hold a name and two children (in reference cells), and whose leaves store data. Here we use integers for simplicity, but in general nodes would hold arbitrary data (e.g., for maps, they would hold key-value pairs):

$$
\begin{aligned}
\text { type trie } & =\text { Nil } \\
& \mid \text { Leaf of int } \\
& \mid \text { Bin of name } *(\text { trie ref }) *(\text { trie ref })
\end{aligned}
$$

The key idea of a probabilistic trie is to use a bit string to identify two things at once: the element stored in the structure (via its hash) and the path to retrieve that element, if it is present. To keep it simple, the code below assumes that all data elements have a unique hash, and that the input trie is complete, meaning that all paths are defined and either terminate in a Nil or a Leaf. Our actual implementation of tries makes neither assumption.

The first operation of a trie is find, which returns either Some data element or None, depending on whether data with the given hash (a list of bools) is present in the trie.

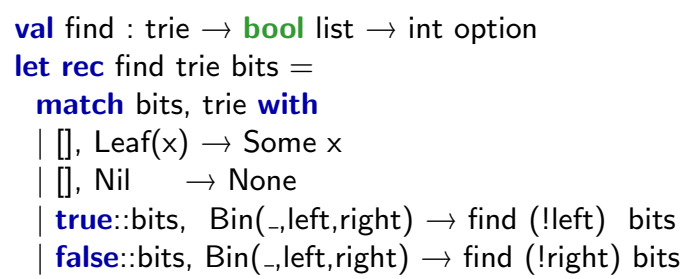

The other operation on tries is extend $n t b d$ which, given an input trie $t$, a data element $d$ and its hash $b$, produces a new trie with $\mathrm{d}$ added to it: 


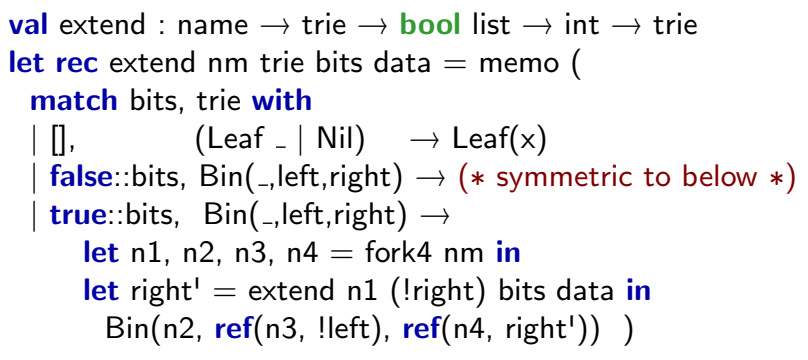

Critically, the first argument to extend is an externally provided $\mathrm{nm}$ that is used to derive names (using fork) for each ref in the new path. Thus, the identity of the trie returned by extend $\mathrm{n} t \mathrm{~b} d$ only depends directly on the name $\mathrm{n}$ and the inserted data, and not on the names or other content of the input trie $t$.

Any incremental program that sequences multiple trie extensions makes critical use of this independence (e.g., the interpreter discussed below). To see how, consider two incremental runs of such a program with two similar sequences of extensions, $[1,2,3,4,5,6]$ versus $[2,3,4,5,6]$, with the same sequence of five names for common elements $[2, \ldots, 6]$. Using the name-based extension above, the tries in both runs will use exactly the same reference cells. By contrast, the structural approach will build entirely new tries in the second run, since the second sequence is missing the leading 1 (a different initial structure). Similarly, using the names in the trie to extend it will also fail here, since it will effectively identify each extension by a global count, which in this case shifts by one for every extension in the second run. Using external names for each extension overcomes both of these problematic behaviors, and gives maximal reuse.

\subsection{Interpreter for IMP}

Finally, as a challenge problem, we used NOMINAL ADAPTON to build an interpreter for IMP (Winskel 1993), a simple imperative programming language. Since our interpreter is incremental, we can efficiently recompute an interpreted program's output after a change to the program code itself. While Adapton requires incrementalized computations to be fully functional, implementing a purely functional interpreter for IMP allows the imperative object language to inherit the incrementality of the meta-language.

The core of our interpreter is a simple, big-step eval function that recursively evaluates an IMP command in some store (that is, a heap) and environment, returning the final store and environment.

Commands include while loops, sequences, conditionals, assignments (of arithmetic and boolean expressions) to variables, and array operations (allocation, reading, and writing). All program values are either booleans or integers. As in $\mathrm{C}$, an integer also doubles as a "pointer" to the store. The interpreter uses finite maps to represent its environment of type env (a mapping from variables to ints) and its store of array content (a mapping from ints to ints).
For incremental efficiency, the interpreter makes critical use of the programming patterns we have seen so far. We use probabilistic tries to represent the finite maps for stores and environments. Each variable assignment or array update in IMP is implemented as a call to extend for the appropriate trie. Names have two uses inside the interpreter. Each call to extend requires a name, as does each recursive call to eval. Classic ADAPTON would identify each with the full structural content of its input. For the IMP language, we require far less information to disambiguate one program state from another.

Consider first the IMP language without while loops. Each subcommand is interpreted at most once; as such, each program state and each path created in the environment or store can be identified with the particular program position of the subcommand being interpreted.

With the addition of while loops, we may interpret a single program position multiple times. To disambiguate these, we thread loop counts through the interpreter represented as a list of integers. The loop count $[3,4]$, for example, tells us we are inside two while loops: the third iteration through the inner loop, within the fourth iteration through the outer loop. We extend the NOMINAL ADAPTON API to allow creating names not just by forking, but also by adding in a counthere, names are created using the loop count paired with the name of the program position. As such, the names sufficiently distinguish recursive calls to the interpreter and paths inside the environment and store.

val eval : $\mathrm{nm} *$ int list $*$ store $*$ env $* \mathrm{cmd} \rightarrow$ store $*$ env

In our experiments, we show that the combination of this naming strategy and probabalistically balanced tries yields an efficient incremental interpreter. Moreover, the interpreter's design provides evidence that NOMINAL ADAPTON's programming patterns are compositional, allowing us to separately choose how to use names for different parts of the program.

\section{Formal Development}

The interaction between memoization, names, and the demanded computation graph is subtle. For this reason, we have distilled NOMINAL ADAPTON to a core calculus called $\lambda_{\text {NomA }}$, which represents the essence of the NOMINAL ADAPTON implementation and formalizes the key algorithms behind incremental computation with names. We prove the fundamental correctness property of incremental computation, dubbed from-scratch consistency, which states that incrementally evaluating a program produces the same answer as re-evaluating it from scratch. This theorem also establishes that (mis)use of names cannot interfere with the meaning of programs; while a poor use of names may have negative impact on performance, it will not cause a program to compute the wrong result. 
We present the full theory but only sketch the consistency result here; the details and proofs are in the extended version of this paper (Hammer et al.|2015).

The formal semantics presented here differs considerably from that of the original ADAPTON system (Hammer et al. 2014). In particular, the original semantics modeled the DCG as an idealized cache of tree-shaped traces that remember all input-output relationships, forever. In contrast, the one developed here models the DCG as it is actually implemented in both ADAPTON and NOMINAL ADAPTON, as a changing graph whose nodes are memoized refs and memoized computations (thunks), and whose edges are their dependencies. Further, via names, this semantics permits more kinds of DCG mutation, since the computations and values of a node can be overwritten via nominal matching. In summary, compared to the prior semantics, our theory more accurately models the implementation, and our metatheory proves correctness in a more expressive setting.

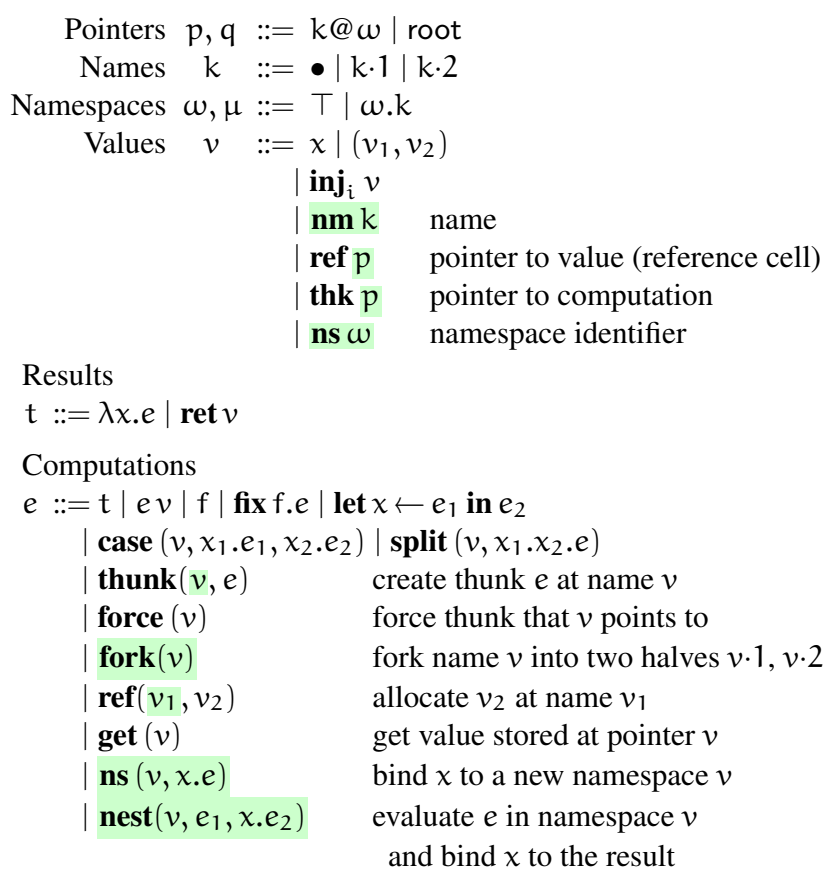

Figure 3: Syntax

\subsection{Syntax}

The syntax of $\lambda_{\text {NomA }}$ is defined in the style of the call-bypush-value (CBPV) calculus (Levy 1999), a standard variant of the lambda calculus with an explicit thunk mechanism. Figure 3 gives the syntax of the language. The nonhighlighted features are standard, and the highlighted forms are new in $\lambda_{\text {NomA }}$.

NOMINAL ADAPTON follows ADAPTON in supporting demand-driven incremental computation using a lazy programming model. In ADAPTON, programmers can write thunk(e) to create a suspended computation, or thunk. The

$$
\begin{aligned}
& \text { Graphs } \\
& \mathrm{G}, \mathrm{H}::=\varepsilon \quad \text { empty graph } \\
& \text { G, p:v p points to value } v \\
& \text { | G, p:e } \quad p \text { points to thunk e (no cached result) } \\
& G, p:(e, t) \quad p \text { points to thunk } e \text { with cached result } t \\
& \mathrm{G},(\mathrm{p}, \mathrm{a}, \mathrm{b}, \mathrm{q}) \mathrm{p} \text { depends on } \mathrm{q} \text { due to action } a \text {, } \\
& \text { with status } b
\end{aligned}
$$

Edge actions

$$
\begin{aligned}
& a::=\quad \text { For edge }(p, a, b, q) \text {, the computation at } p . . \\
& \text { alloc } v \text {... created reference } v \text { at } \mathrm{q} \\
& \text { alloc } e \text {...created thunk } e \text { at } q \\
& \text { obs } v \ldots \text { read q's value, which was } v \\
& \text { | obs t ...forced thunk q, which returned } t
\end{aligned}
$$

Edge statuses

$\mathrm{b}::=$ clean value or computation at sink is out of date

| dirty value or computation at sink is up to date

Figure 4: Graphs

thunk v's value is computed only when it is forced, using syntax force $(v)$. Thunks also serve as ADAPTON's (and NOMINAL ADAPTON's) unit of incremental reuse: if we want to reuse a computation, we must make a thunk out of it. The syntax memo(e) we used earlier is shorthand for force $(\operatorname{thunk}(e, e))$, where we abuse notation and treat $e$ as a name that identifies itself 3 This construction introduces a thunk that the program immediately forces, eliminating laziness, but supporting memoization.

CBPV distinguishes values, results (or terminal computations), and computations. A computation e can be turned into a value by thunking it via thunk $(v, e)$. The first argument, the name $v$, is particular to NOMINAL ADAPTON; ordinary CBPV does not explicitly name thunks. Conversely, a value $v$ can play the role of a result $t$ via ret $v$; results $t$ are a subclass of computations $e$.

Functions $\lambda x . e$ are terminal computations; $e v$ evaluates $e$ to a function $\lambda x \cdot e^{\prime}$ and substitutes $v$ for $e^{\prime}$. Note that the function argument in $e v$ is a value, not a computation.

Let-expressions let $x \leftarrow e_{1}$ in $e_{2}$ evaluate the computation $e_{1}$ first. The usual $\lambda$-calculus application $e_{1} e_{2}$ can be simulated by let $x \leftarrow e_{2}$ in $\left(e_{1} x\right)$. Fixed points fix f.e are computations, and so are fixed point variables $f$.

Given an injection into a disjoint union, $\mathbf{i n j}_{i} v$, the case computation form eliminates the sum and computes the corresponding $e_{i}$ branch, with $v$ substituted for $x_{i}$. Given a pair $\left(v_{1}, v_{2}\right)$, the computation split $\left(v, x_{1} . x_{2} . e\right)$ computes $e$, first substituting $v_{1}$ for $x_{1}$, and $v_{2}$ for $x_{2}$.

For more on (non-nominal, non-incremental) formulations of CBPV, including discussion of value types and computation types, see Levy (1999, 2001).

Graphs, Pointers, and Names. Graphs G are defined in Figure 4. They represent the mutable store (references),

\footnotetext{
${ }^{3}$ Notice that NOMINAL ADAPTON thunks are also named, which provides greater control over reuse.
} 
memo tables (which cache thunk results), and the DCG. Element $p: v$ says that pointer p's current value is $v$. Element p:e says that pointer $p$ is the name of thunk $e$. Element $p:(e, t)$ says that $p$ is the name of thunk $e$ with a previously computed result $t$ attached. Element $(p, a, b, q)$ is a DCG edge indicating that the thunk pointed to by $p$ depends on node $q$, where $q$ could either name another thunk or a reference cell. Dependency edges also reflect the action a that produced the edge and the edge's status b (whether it is clean or dirty).

Pointers in $\lambda_{\text {NomA }}$ are represented as pairs $k @ \omega 4^{4}$ where nm $k$ was the name given as the first argument in a call to thunk or ref, and $\omega$ was the namespace in which the call to thunk or ref took place. This namespace $\omega$ is either $T$ for the top-level, or some $\mu . k_{1}$ as set by a call to nest. For the latter case, the program would first construct a value ns $\mu . k_{1}$ by calling $\mathbf{n s}$ with first argument $\mathbf{n m ~} \mathrm{k}_{1}$ while in namespace $\mu$. Notice that pointers and namespaces have similar structure, and similar assurances of determinism when creating named thunks, references, and namespaces. Finally, names $k$ consist of the root name $\bullet$, while other names are created by "forking" existing names: invoking fork $(\mathbf{n m ~ k})$ produces names $\mathbf{n m ~ k \cdot 1 ~ a n d ~} \mathbf{n m} \mathrm{k} \cdot 2$.

\subsection{Semantics}

We define a big-step operational semantics (Figure 5) with judgments $G_{1} \vdash_{\omega}^{p}$ e $\Downarrow G_{2} ; t$, which states that under input graph $G_{1}$ and within namespace $\omega$, evaluating the computation e produces output graph $\mathrm{G}_{2}$ and result $t$, where $e$ 's evaluation was triggered by a previous force of thunk $p$.

Incremental and Reference Systems. In order to state and later prove our central meta-theoretic result, from-scratch consistency, we define two closely-related systems of evaluation rules: an incremental system and a non-incremental or reference system. The incremental system models NoMINAL ADAPTON programs that transform a graph whose nodes are store locations (values and thunks) and whose edges represent dependencies (an edge from $p$ to $q$ means that $q$ depends on $p$ ); the reference system models call-bypush-value programs under a plain store (a graph with no edges). Since it has no IC mechanisms, everything the reference system does is, by definition, from-scratch consistent.

Rules above the double horizontal line in Figure 5 do not manipulate the graph, and are common to the incremental and reference systems. The shaded rules, to the left of vertical double lines, are non-incremental rules that never create edges and do not cache results. The rules to their right create edges, store cached results, and recompute results that have become invalid.

\subsubsection{Common Rules}

Several of the rules at the top of Figure 5 are derived from standard CBPV rules.

\footnotetext{
${ }^{4}$ The pointer root is needed to represent the top-level "thunk" in the semantics, but will never be mapped to an actual value or expression.
}

Rules for standard language features (pairs, sums, functions, fix, and let) straightforwardly adapt the standard rules, ignoring $p$ and $\omega$ and "threading through" input and output graphs. For example, Eval-app evaluates a function $e_{1}$ to get a terminal computation $\lambda x . e_{2}$ and substitutes the argument $v$ for $x$, threading through the graph: evaluating $e_{1}$ produces $\mathrm{G}_{2}$, which is given as input to the second premise, resulting in output graph $\mathrm{G}_{3}$. Rule Eval-case, applying case to a sum inj $_{i} v$, substitutes $v$ for $x_{i}$ in the appropriate case arm.

The last three shared rules are not standard: they deal with names and namespaces.

- Eval-fork splits a name $k$ into children $k \cdot 1$ and k.2. Once forked, the name $k$ should not be used to allocate a new reference or thunk, nor should $k$ be forked again.

- Running in namespace $\omega$, Eval-namespace makes a new namespace $\omega . k$ and substitutes it for $x$ in the body $e$.

- Running in namespace $\omega$, Eval-nest runs $e_{1}$ in a different namespace $\mu$ and then returns to $\omega$ to run $e_{2}$, with $x$ replaced by the result of running $e_{1}$.

\subsubsection{Non-Incremental Rules}

These rules cover allocation and use of references and thunks. Like the incremental rules (discussed below), they use names and namespaces; however, they do not cache the results of thunks. We discuss the non-incremental rules first, because they are simpler and provide a kind of skeleton for the incremental rules.

- Eval-refPlain checks that the pointer described by $\mathrm{q}=$ $k @ \omega$ is fresh ( $q \notin \operatorname{dom}\left(\mathrm{G}_{1}\right)$ ), adds a node $q$ with contents $v$ to the graph $\left(\mathrm{G}_{1}\{\mathbf{q} \mapsto v\}=\mathrm{G}_{2}\right)$, and returns a reference ref $q$.

- Eval-thunkPlain is similar to Eval-refPlain, but creates a node with a suspended computation $e$ instead of a value.

- Eval-getPlain returns the contents of the pointer $q$.

- Eval-forcePlain extracts the computation stored in a thunk $\left(\mathrm{G}_{1}(\mathrm{q})=\mathrm{e}\right)$ and evaluates it under the namespace of $q$; that is, if $q=k @ \mu$, it evaluates it under $\mu$. (The rule uses namespace $(k @ \mu)=\mu$ ).

\subsubsection{Incremental Rules}

Each non-incremental rule corresponds to one or more incremental rules: the incremental semantics is influenced by the graph edges, which are not present in the non-incremental system. For example, Eval-refPlain is replaced by EvalrefDirty and Eval-refClean.

These rules use some predicates and operations, such as dirty-paths-in, that we explain informally as we describe the rules; they are fully defined in Figure 6 .

Incremental computation arises by making $\mathrm{G}_{1}$ a modification of a previously produced graph $G_{2}$, and then rerunning e. A legal modification involves replacing references $p: v$ with $p: v^{\prime}$ and dirtying all edges along paths to $p$ in 
$\mathrm{G}_{1} \vdash_{\omega}^{\mathrm{p}} e \Downarrow \mathrm{G}_{2} ; \mathrm{t}$ Under graph $\mathrm{G}_{1}$, evaluating expression $e$ as part of thunk $p$ in namespace $\omega$ yields $\mathrm{G}_{2}$ and $t$.

Rules common to the non-incremental and incremental systems:

$$
\begin{aligned}
& \begin{array}{c}
\mathrm{G}_{1} \vdash_{\omega}^{\mathrm{p}} e_{1} \Downarrow \mathrm{G}_{2} ; \lambda x_{.} e_{2} \\
\overline{\mathrm{G} \vdash{ }_{\omega}^{\mathrm{p}} \mathrm{t} \Downarrow \mathrm{G} ; \mathrm{t}} \text { Eval-term } \quad \frac{\mathrm{G}_{2} \vdash_{\omega}^{p}[v / x] e_{2} \Downarrow \mathrm{G}_{3} ; \mathrm{t}}{\mathrm{G}_{1} \vdash_{\omega}^{\mathrm{p}} e_{1} v \Downarrow \mathrm{G}_{3} ; \mathrm{t}} \text { Eval-app }
\end{array} \\
& \mathrm{G}_{1} \vdash_{\omega}^{\mathrm{p}} \mathrm{e}_{1} \Downarrow \mathrm{G}_{2} ; \operatorname{ret} v \\
& \frac{\mathrm{G}_{1} \vdash_{\omega}^{\mathrm{p}}[(\mathbf{f i x} \text { f.e }) / \mathrm{f}] e \Downarrow \mathrm{G}_{2} ; \mathrm{t}}{\mathrm{G}_{1} \vdash_{\omega}^{\mathrm{p}} \text { fixf.e } \Downarrow \mathrm{G}_{2} ; \mathrm{t}} \text { Eval-fix } \quad \frac{\mathrm{G}_{2} \vdash_{\omega}^{\mathrm{p}}[v / x] e_{2} \Downarrow \mathrm{G}_{3} ; t}{\mathrm{G}_{1} \vdash_{\omega}^{\mathrm{p}} \text { let } x \leftarrow e_{1} \text { in } e_{2} \Downarrow \mathrm{G}_{3} ; \mathrm{t}} \text { Eval-bind } \\
& \frac{\mathrm{G}_{1} \vdash_{\omega}^{\mathrm{p}}\left[v / x_{i}\right] e_{i} \Downarrow \mathrm{G}_{2} ; \mathrm{t}}{\mathrm{G}_{1} \vdash_{\omega}^{\mathrm{p}} \operatorname{case}\left(\mathbf{i n j}_{\mathrm{i}} v, \mathrm{x}_{1} . e_{1}, \mathrm{x}_{2} . e_{2}\right) \Downarrow \mathrm{G}_{2} ; \mathrm{t}} \text { Eval-case } \frac{\mathrm{G}_{1} \vdash_{\omega}^{\mathrm{p}}\left[v_{1} / \mathrm{x}_{1}\right]\left[v_{2} / \mathrm{x}_{2}\right] e \Downarrow \mathrm{G}_{2} ; \mathrm{t}}{\mathrm{G}_{1} \vdash_{\omega}^{\mathrm{p}} \text { split }\left(\left(v_{1}, v_{2}\right), \mathrm{x}_{1} \cdot \mathrm{x}_{2} . e\right) \Downarrow \mathrm{G}_{2} ; \mathrm{t}} \text { Eval-split } \\
& \mathrm{G}_{\omega_{\omega}}^{\mathrm{p}} \operatorname{fork}(\mathbf{n m k}) \Downarrow \mathrm{G} ; \operatorname{ret}(\mathbf{n m} k \cdot 1, \mathbf{n m} k \cdot 2)
\end{aligned}
$$

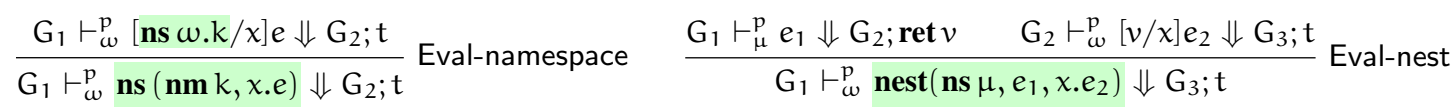

Rules specific to the (non-incremental || incremental) systems:

$$
\begin{aligned}
& \mathrm{q}=\mathrm{k} @ \omega \\
& \frac{\mathrm{q} \notin \operatorname{dom}\left(\mathrm{G}_{1}\right) \quad \mathrm{G}_{1}\{\mathrm{q} \mapsto v\}=\mathrm{G}_{2}}{\mathrm{G}_{1} \vdash_{\omega}^{\mathrm{p}} \operatorname{ref}(\mathbf{n m} k, v) \Downarrow \mathrm{G}_{2} ; \text { ret ref } \mathrm{q}} \text { Eval-refPlain }
\end{aligned}
$$

$$
\frac{q=k @ \omega}{G_{1} \vdash{ }_{\omega}^{p} \operatorname{ref}(\mathbf{n m k} k v) \Downarrow G_{3},(p, \text { alloc } v \text {, clean, } q) ; \text { ret ref } q} \text { Eval-refDirty }
$$

$$
\frac{\mathrm{q}=\mathrm{k} @ \omega \quad \mathrm{G}(\mathrm{q})=v}{\mathrm{G} \vdash_{\omega}^{\mathrm{p}} \operatorname{ref}(\mathbf{n m} k, v) \Downarrow \mathrm{G},(\mathrm{p}, \text { alloc } v, \text { clean, } \mathrm{q}) ; \operatorname{ret} \operatorname{ref} \mathrm{q}} \text { Eval-refClean }
$$

$\frac{q=k @ \omega \quad G_{1}\{q \mapsto e\}=G_{2} \quad \operatorname{dirty}-\text { paths-in }\left(G_{2}, q\right)=G_{3}}{G_{1} \vdash_{\omega}^{p} \text { thunk }(\text { nmk } k, e) \Downarrow G_{3},(p, \text { alloc } e, \text { clean, } q) ; \operatorname{ret}(\text { thk } q)}$ Eval-thunkDirty

$q=k @ \omega$

$\mathrm{q} \notin \operatorname{dom}\left(\mathrm{G}_{1}\right) \quad \mathrm{G}_{1}\{\mathrm{q} \mapsto e\}=\mathrm{G}_{2}$

$\mathrm{G}_{1} \vdash_{\omega}^{\mathrm{p}} \operatorname{thunk}(\mathbf{n m k}, e) \Downarrow \mathrm{G}_{2} ;$ ret $($ thk q $)$ Eval-thunkPlai

$$
\frac{\mathrm{q}=\mathrm{k} @ \omega \quad \exp (\mathrm{G}, \mathrm{q})=e}{\mathrm{G} \vdash_{\omega}^{\mathrm{p}} \operatorname{thunk}(\mathbf{n m} k, e) \Downarrow \mathrm{G},(\mathrm{p}, \text { alloc } e, \text { clean, } \mathrm{q}) ; \text { ret }(\text { thk } \mathrm{q})} \text { Eval-thunkClean }
$$

$$
\frac{\mathrm{G}(\mathrm{q})=v}{\mathrm{G} \vdash_{\omega}^{\mathrm{p}} \operatorname{get}(\operatorname{ref} \mathrm{q}) \Downarrow \mathrm{G} ; \boldsymbol{r e t} v} \text { Eval-getPlain } \| \frac{\mathrm{G}(\mathrm{q})=v}{\mathrm{G} \vdash_{\omega}^{\mathrm{p}} \text { get }(\operatorname{ref} \mathrm{q}) \Downarrow \mathrm{G},(\mathrm{p}, \mathbf{o b s} v, \text { clean, } \mathrm{q}) ; \boldsymbol{r e t} v} \text { Eval-getClean }
$$

$$
\begin{aligned}
& \mathrm{G}_{1}(\mathrm{q})=e \\
& \frac{\mathrm{G}_{1} \vdash_{\text {namespace }(\mathrm{q})}^{\mathrm{q}} \mathrm{e} \Downarrow \mathrm{G}_{2} ; \mathrm{t}}{\mathrm{G}_{1} \vdash_{\omega}^{\mathrm{p}} \text { force }(\text { thk } \mathrm{q}) \Downarrow \mathrm{G}_{2} ; \mathrm{t}} \text { Eval-forcePlain }
\end{aligned}
$$

$$
\begin{aligned}
& \frac{\mathrm{G}(\mathrm{q})=(\mathrm{e}, \mathrm{t}) \quad \text { all-clean-out }(\mathrm{G}, \mathrm{q})}{\mathrm{G} \vdash_{\omega}^{\mathrm{p}} \text { force }(\text { thk } \mathrm{q}) \Downarrow \mathrm{G},(\mathrm{p}, \text { obs } \mathrm{t}, \text { clean, } \mathrm{q}) ; \mathrm{t}} \text { Eval-forceClean } \\
& \text { all-clean-out }\left(\left(\mathrm{G}_{1}, \mathrm{G}_{2}\right), \mathrm{q}_{2}\right) \\
& \text { consistent-action }\left(\left(\mathrm{G}_{1}, \mathrm{G}_{2}\right), \mathrm{a}, \mathrm{q}_{2}\right) \\
& \frac{G_{1},\left(q_{1}, a \text {, clean, } q_{2}\right), G_{2} \vdash{ }_{\omega}^{p} \text { force }\left(\text { thk } p_{0}\right) \Downarrow G_{3} ; t}{G_{1},\left(q_{1}, a, \text { dirty, } q_{2}\right), G_{2} \vdash_{\omega}^{p} \text { force }\left(\text { thk } p_{0}\right) \Downarrow G_{3} ; t} \text { Eval-scrubEdge } \\
& \exp \left(\mathrm{G}_{1}, \mathrm{q}\right)=\mathrm{e}^{\prime} \quad \mathrm{G}_{2}\left\{\mathrm{q \mapsto}\left(\mathrm{e}^{\prime}, \mathrm{t}^{\prime}\right)\right\}=\mathrm{G}_{2}^{\prime} \\
& \text { del-edges-out }\left(\mathrm{G}_{1}\left\{\mathrm{q} \mapsto \mathrm{e}^{\prime}\right\}, \mathrm{q}\right)=\mathrm{G}_{1}^{\prime} \quad \text { all-clean-out }\left(\mathrm{G}_{2}^{\prime}, \mathrm{q}\right) \\
& \frac{\left.\mathrm{G}_{1}^{\prime} \vdash_{\text {namespace }(\mathrm{q})}^{\mathrm{q}} \mathrm{e}^{\prime} \Downarrow \mathrm{G}_{2} ; \mathrm{t}^{\prime} \quad \mathrm{G}_{2}^{\prime} \vdash_{\omega}^{\mathrm{p}} \text { force (thk } \mathrm{p}_{0}\right) \Downarrow \mathrm{G}_{3} ; \mathrm{t}}{\mathrm{G}_{1} \vdash_{\omega}^{\mathrm{p}} \text { force }\left(\text { thk } \mathrm{p}_{0}\right) \Downarrow \mathrm{G}_{3} ; \mathrm{t}} \text { Eval-computeDep }
\end{aligned}
$$

Figure 5: Evaluation rules of $\lambda_{\text {NomA }}$; vertical bars separate non-incremental rules (left, shaded) from incremental rules (right) 


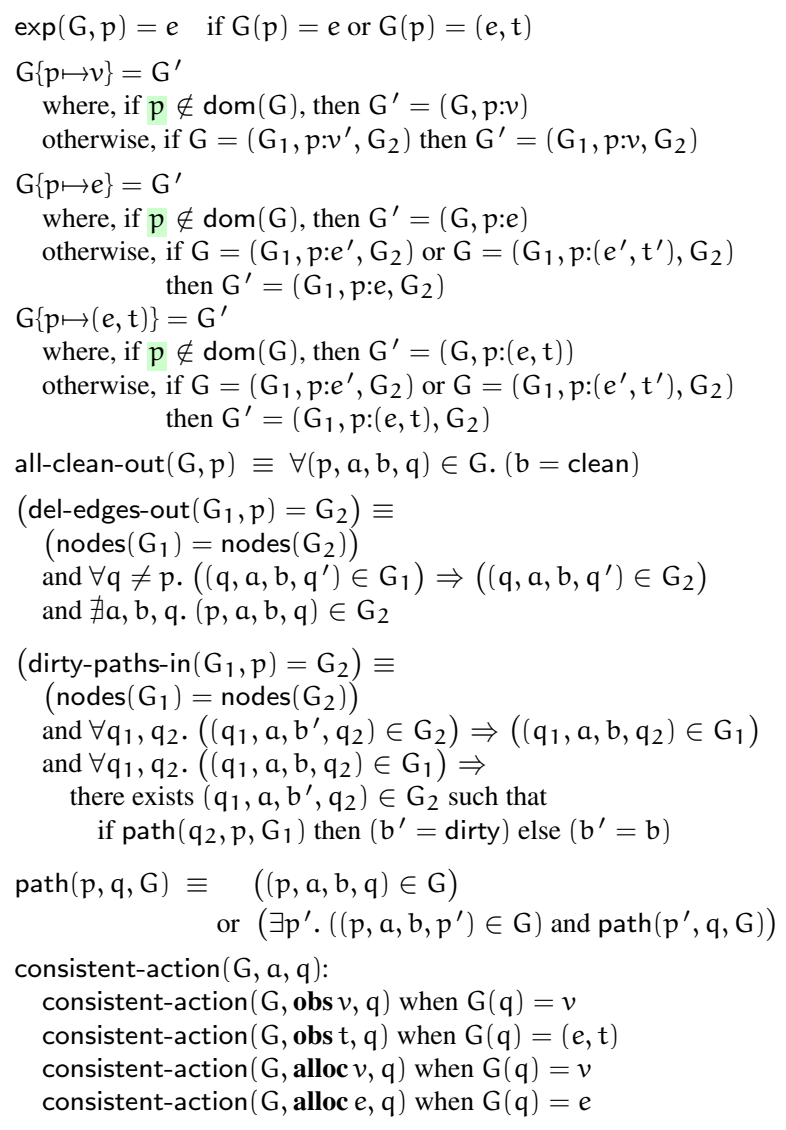

Figure 6: Graph predicates and transformations

the DCG: dirty-paths-in $\left(G_{1}, p\right)$ is the same as $G_{1}$ but with edges on paths to $p$ marked dirty.

Creating Thunks. The DCG is constructed during evaluation. The main rule for creating a thunk is Eval-thunkDirty, which converts computation $e$ into a thunk by generating a pointer $q$ from the provided name $k$, which couples the name with the current namespace $\omega$. The output graph is updated to map $q$ to $e$. If $q$ happens to be in the graph already, all paths to it will be dirtied. Finally, the rule adds edge ( $p$, alloc $e$, clean, $q)$ to the output graph, indicating that the currently evaluating thunk $p$ depends on $q$ and is currently clean.

Forcing Thunks. Forcing a thunk that has not been previously computed, an operation that involves one rule in the non-incremental system (Eval-forcePlain), involves at least two rules in the incremental system: Eval-computeDep and Eval-forceClean.

Rule Eval-forceClean performs memoization: Given q pointing to $(e, t)$, where $t$ is the cached result of the thunk $e$, if q's outgoing edges are clean then $t$ is consistent and can be reused. Thus Eval-forceClean returns $t$ immediately without reevaluating $e$, but adds an edge denoting that $p$ has observed the result of $q$ to be $t$.
Rule Eval-computeDep applies when $\boldsymbol{e}=$ force $\left(\right.$ thk $\left.\mathrm{p}_{0}\right)$. This rule serves two purposes: it forces thunks for the first time, and it selectively recomputes until a cached result can be reused.

Its first premise $\exp \left(\mathrm{G}_{1}, \mathrm{q}\right)=e^{\prime}$ nondeterministically chooses some thunk $q$ whose suspended expression is $e^{\prime}$ (whether or not $q$ also has a cached result). Its second premise del-edges-out $\left(\mathrm{G}_{1}\left\{\mathbf{q} \mapsto e^{\prime}\right\}, \mathbf{q}\right)=\mathrm{G}_{1}^{\prime}$ updates $\mathrm{G}_{1}$ so that $q$ points to $e^{\prime}$ (removing q's cached result, if any), and deletes outward edges of $q$. We need to delete the outward edges before evaluating $e^{\prime}$ because they represent what a previous evaluation of $e^{\prime}$ depended on. The third premise recomputes q's expression $e^{\prime}$, with $q$ as the current thunk and q's namespace component as the current namespace. In the fourth premise, the recomputed result $t^{\prime}$ is cached, resulting in graph $\mathrm{G}_{2}^{\prime}$.

The final premise evaluates force $\left(\right.$ thk $\left.p_{0}\right)$, the same expression as the conclusion, but under a graph $\mathrm{G}_{2}^{\prime}$ containing the result of evaluating $e^{\prime}$. In deriving this premise we may again apply Eval-computeDep to "fix up" other nodes of the graph, but will eventually end up with the thunk $q$ chosen in the first premise being $p_{0}$ itself. In this case, the last premise of Eval-computeDep will be derived by EvalforceClean (with $q$ instantiated to $p_{0}$ ).

We skipped the fifth premise all-clean-out $\left(G_{2}^{\prime}, q\right)$, which demands that all outgoing edges from $q$ in the updated graph are clean. This consistency check ensures that the program has not used the same name for two different thunks or references, e.g., by calling thunk $\left(\mathbf{n m k}, e_{1}\right)$ and later $\operatorname{thunk}\left(\mathbf{n m k}, e_{2}\right)$ in the same namespace $\omega$. If this happens, the graph will first map $k @ \omega$ to $e_{1}$ but will later map it to $e_{2}$. Without this check, a computation $q$ that depends on both $e_{1}$ and $e_{2}$ could be incorrect, because (re-)computing one of them might use cached values that were due to the other. Fortunately, this potential inconsistency is detected by all-clean-out: When a recomputation of q results in $k @ \omega$ being mapped to a different value, all existing paths into $k @ \omega$ are dirtied (by the last premise of Eval-thunkDirty above). Since $q$ is one of the dependents, it will detect that fact and can signal an error.

This fifth premise formalizes the double-use checking algorithm first described in Section 2.4. In particular, each use of Eval-computeDep corresponds to the implementation pushing (and later popping) a node from its force stack. By inspecting the outgoing edges upon each pop, it effectively verifies that each node popped from the stack is clean.

Note that in the case $q=p_{0}$-where the "dependency" being computed is $p_{0}$ itself- the last premise could be derived by Eval-forceClean, which looks up the result just computed by $\mathrm{G}_{1}^{\prime} \vdash_{\text {namespace }(\mathrm{q})}^{\mathrm{q}} \mathrm{e}^{\prime} \Downarrow \mathrm{G}_{2} ; \mathrm{t}^{\prime}$.

Replacing Dirty Edges with Clean Edges. Eval-scrubEdge replaces a dirty edge $\left(q_{1}, a\right.$, dirty, $\left.q_{2}\right)$ with a clean edge $\left(q_{1}, a\right.$, clean, $\left.q_{2}\right)$. First, it checks that all edges out from $q_{2}$ are clean; this means that the contents of $q_{2}$ are up-to- 
date. Next, it checks that the action a that represents $q_{1}$ 's dependency on $q_{2}$ is consistent with the contents of $q_{2}$. For example, if $q_{2}$ points to a thunk with a cached result $\left(e_{2}, t_{2}\right)$ and $a=$ obs $t$, then the "consistent-action" premise checks that the currently cached result $t_{2}$ matches the result $t$ that was previously used by $\mathrm{q}_{1}$.

Creating Reference Nodes. Like Eval-refPlain, Eval-refDirty creates a node $q$ with value $v: G_{1}\{q \mapsto v\}=G_{2}$. Unlike Eval-refPlain, Eval-refDirty does not check that $q$ is not in the graph: if we are recomputing, $q$ may already exist. So $\mathrm{G}_{1}\{\mathrm{q} \mapsto v\}=\mathrm{G}_{2}$ either creates $\mathrm{q}$ pointing to $v$, or updates $\mathrm{q}$ by replacing its value with $\nu$. It then marks the edges along all paths into $q$ as dirty dirty-paths-in $\left(G_{2}, q\right)=G_{3}$; these are the paths from nodes that depend on $q$.

Re-creating Clean References. Eval-refClean can be applied only during recomputation, and only when $\mathrm{G}(\mathrm{q})=v$. That is, we are evaluating $\operatorname{ref}(\mathbf{n m k}, v)$ and allocating the same value as the previous run. Since the values are the same, we need not mark any dependency edges as dirty, but we do add an edge to remember that $p$ depends on $q$.

Creating Thunks. Eval-thunkDirty corresponds exactly to Eval-refDirty, but for thunks rather than values.

Re-creating Thunks. Eval-thunkClean corresponds to EvalrefClean and does not change the contents of $q$. Note that the condition $(\exp (G, q))=e$ applies whether or not $q$ includes a cached result. If a cached result is present, that is, $\mathrm{G}(\mathrm{q})=(e, \mathrm{t})$, it remains in the output graph.

Reading References. Eval-getClean is the same as EvalgetPlain, except that it adds an edge representing the dependency created by reading the contents of $q$.

The Rules vs. the Implementation. Our rules are not intended to be an "instruction manual" for building an implementation; rather, they are intended to model our implementation. To keep the rules simple, we underspecify two aspects of the implementation.

First, when recomputing an allocation in the case when the allocated value is equal to the previously allocated value $(\mathrm{G}(\mathrm{q})=v)$, either Eval-refDirty or Eval-refClean applies. However, in this situation the implementation always follows the behavior of Eval-refClean, since that is the choice that avoids unnecessarily marking edges as dirty and causing more recomputation. An analogous choice exists for EvalthunkClean versus Eval-thunkDirty.

Second, similarly to the original ADAPTON system, the timing of dirtying and cleaning is left open: Eval-scrubEdge can be applied to dirty edges with no particular connection to the $p_{0}$ mentioned in the subject expression force (thk $\left.p_{0}\right)$, and the timing of recomputation via Eval-computeDep is left open as well. Our implementations of dirtying and reevaluation fix these open choices, and they are each analogous to the algorithms found in the original ADAPTON work.
For details, we refer the interested reader to Algorithm 1 in Hammer et al. (2014).

\subsection{From-Scratch Consistency}

We show that an incremental computation modeled by our evaluation rules has a corresponding non-incremental computation: given an incremental evaluation of $e$ that produced $t$, a corresponding non-incremental evaluation also produces t. Moreover, the values and expressions in the incremental output graph match those in the graph produced by the nonincremental evaluation.

Eliding some details and generalizations, the from-scratch consistency result is:

Theorem. If incremental $\mathcal{D}_{i}$ derives $\mathrm{G}_{1} \vdash_{\omega}^{\mathrm{p}} \mathrm{e} \Downarrow \mathrm{G}_{2} ; \mathrm{t}$, then a non-incremental $\mathcal{D}_{n i}$ derives $\left\lfloor\mathrm{G}_{1}\right\rfloor_{\mathrm{P}_{1}} \vdash_{\omega}^{\mathrm{p}} \mathrm{e} \Downarrow\left\lfloor\mathrm{G}_{2}\right\rfloor_{\mathrm{P}_{2}} ; \mathrm{t}$ where $\left\lfloor\mathrm{G}_{1}\right\rfloor_{\mathrm{P}_{1}} \subseteq\left\lfloor\mathrm{G}_{2}\right\rfloor_{\mathrm{P}_{2}}$ and $\mathrm{P}_{2}=\mathrm{P}_{1} \cup \operatorname{dom}(W)$.

Here, $W$ is the set of pointers that $\mathcal{D}_{i}$ may allocate. The restriction function $\left\lfloor\mathrm{G}_{i}\right\rfloor_{\mathrm{P}_{i}}$ drops all edges from $\mathrm{G}_{i}$ and keeps only nodes in the set $P_{i}$. It also removes any cached results $t$. The set $P_{i}$ corresponds to the nodes in $G_{i}$ that are present at this point in the non-incremental derivation, which may differ from the incremental derivation since EvalcomputeDep need not compute dependencies in left-to-right order.

The full statement, along with definitions of $W$, the restriction function, and lemmas, is in the extended version (Hammer et al. 2015) as Theorem B.13.

\section{Implementation}

We implemented NOMINAL ADAPTON as an OCaml library. In this section, we describe its programming interface, data structures, and algorithms. Additional details about memory management appear in Appendix A. The code for NOMINAL ADAPTON is freely available:

https://github.com/plum-umd/adapton.ocaml

\subsection{Programming Interface}

Figure 7 shows the basic Nominal ADAPTON API. Two of the data types, name and aref, correspond exactly to names and references in Sections 2 and 4 The other data types, $\mathrm{mfn}$ and athunk, work a little differently, due to limitations of OCaml: In OCaml, we cannot type a general-purpose memo table (containing thunks with non-uniform types), nor can we examine a thunk's "arguments" (that is, the values of the variables in a closure's environment) 5

To overcome these limitations, our implementation creates a tight coupling between namespaces and memoized functions. The function mk_mfn $k f$ takes a name $k$ and a function $f$ and returns a memoized function $\mathrm{mfn}$. The function $\mathrm{f}$ must have type ('arg,'res) mbody, i.e., it takes an $\mathrm{mfn}$

\footnotetext{
${ }^{5}$ Recall from the start of the previous section that memoized calls are implemented as thunks in NOMINAL ADAPTON.
} 


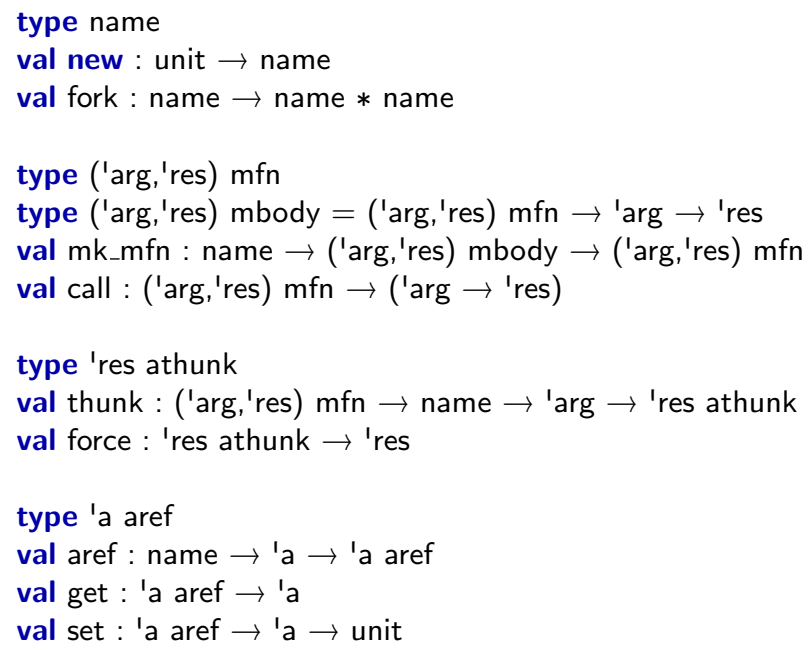

Figure 7: Basic NominAl ADAPTON API

and an 'arg as arguments, and produces a 'res. (The $\mathrm{mfn}$ is for recursive calls; see the example below.)

Later on, we call thunk $\mathrm{m} \mathrm{k}$ arg to create a thunk of type athunk from the memoized function $m$, with thunk name $k$ (relative to m's namespace) and argument arg. The code for the thunk will be whatever function $m$ was created from. In other words, in our implementation, only function calls can be memoized (not arbitrary expressions), and each set of thunks that share the same function body also share the same namespace.

Using this API, we can rewrite the map code from Section 2.3 as follows:

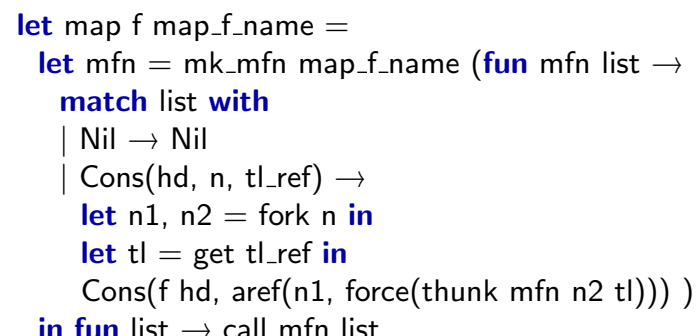

The code above differs from the earlier version in that the programmer uses mk_mfn with the name map_f_name to create a memo table in a fresh namespace. Moreover, memoization happens directly on the recursive call, by introducing a thunk (and immediately forcing it).

\subsection{Implementing Reuse}

Much of the implementation of NOMINAL ADAPTON remains unchanged from classic ADAPTON. Specifically, both systems use DCGs to represent dependency information among nodes representing thunks and refs, and both systems traverse their DCGs to dirty dependencies and to later reuse (and repair) partially inconsistent graph components.
These steps were described in Sections 2 and 4 and were detailed further by Hammer et al. (2014).

The key differences between NOMINAL ADAPTON and Classic ADAPTON have to do with memo tables and thunks.

Memo Tables and Thunks. NOMINAL ADAPTON memo tables are implemented as maps from names to DCG nodes, which contain the thunks they represent. When creating memo tables with mk_mfn, the programmer supplies a name and an mbody. Using the name, the library checks for an existing table (i.e., a namespace). If none exists, it creates an empty table, registers it globally, and returns it as an $\mathrm{mfn}$. If a table exists, then the library checks that the given mbody is (physically) equal to the mbody component of the existing $\mathrm{mfn}$; it issues a run-time error if not.

When the program invokes thunk, it provides an mfn, name, and argument. The library checks the mfn's memo table for an existing node with the provided name. If none exists, it registers a fresh node with the given name in the memo table and adds an allocation edge to it from the current node (which is set whenever a thunk is forced).

If a node with the same name already exists, the library checks whether the argument is equal to the current one. If equal, then the thunk previously associated with the name is the same as the new thunk, so the library reuses the node, returning it as an athunk and adding an allocation edge to the DCG. If not equal, then the name has been allocated for a different thunk either in a prior run, or in this run. The latter case is an error that we detect and signal. To distinguish these two cases, we use the check described in Section 2.4. Assuming no error, the library needs to reset the state associated with the name: It clears any prior cached result, dirties any incoming edges (transitively), mutates the argument stored in the node to be the new one, and adds an allocation edge. Later, when and if this thunk is forced, the system will run it. Further, because of the dirtying traversal, any nodes that (transitively or directly) forced this changed node are also candidates for reevaluation.

Names. A name in NOMINAL ADAPTON is implemented as a kind of list, as follows:

type name $=$ Bullet $\mid$ One of int $*$ name $\mid$ Two of int $*$ name

Ignoring the int part, this is a direct implementation of

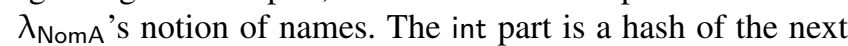
element in the list (but not beyond it), to speed up disequality checks - if two One or Two elements do not share the same hash field they cannot be equal; if they do, we must compare their tails (because of hash collisions). Thus, at worst, establishing equality is linear in the length of the name, but we can short circuit a full traversal in many cases. We note that in our applications, the size of names is either a constant, or it is proportional to the depth (not total size) of the DCG, which is usually sublinear (e.g., logarithmic) in the current input's total size. 


\section{Experimental Results}

This section evaluates NOMINAL ADAPTON's performance against ADAPTON and from-scratch recomputation 6 We find that NOMINAL ADAPTON is nearly always faster than ADAPTON, which is sometimes orders of magnitude slower than from-scratch computation. NOMINAL ADAPTON always enjoys speedups, and sometimes very dramatic ones (up to $10900 \times$ ).

\subsection{Experimental Setup}

Our experiments measure the time taken to recompute the output of a program after a change to the input, for a variety of different sorts of changes. We compare NOMINAL ADAPTON against classic ADAPTON and from-scratch computation on the changed input; the latter avoids all IC-related overhead and therefore represents the best from-scratch time possible.

We evaluate two kinds of subject programs. The first set is drawn from the IC literature on SAC and Adapton (Hammer et al. 2011, 2009, 2014). These consist of standard list processing programs: (eager and lazy) filter, (eager and lazy) map, reduce( $\min )$, reduce(sum), reverse, median, and a listbased mergesort algorithm. Each program operates over randomly generated lists. These aim to represent key primitives that are likely to arise in standard functional programs, and use the patterns discussed in Section 3.1. We also consider an implementation of quickhull (Barber et al. 1996), a divideand-conquer method for computing the convex hull of a set of points in a plane. Convex hull has a number of applications including pattern recognition, abstract interpretation, computational geometry, and statistics.

We also evaluate an incremental IMP interpreter, as discussed in Section 3.3 . measuring its performance on a variety of different IMP programs. fact iteratively computes the factorial of an integer. intlog;fact evaluates the sequence of computing an integer logarithm followed by factorial. array max allocates, initializes, and destructively computes the maximum value in an array. matrix mult allocates, initializes, and multiplies two square matrices (implemented as arrays of arrays of integers). These IMP programs exhibit imperative behavior not otherwise incrementalizable, except as programs evaluated by a purely functional, big-step interpreter implemented in an incremental meta-language.

All programs were compiled using OCaml 4.01.0 and run on an 8-core, $2.26 \mathrm{GHz}$ Intel Mac Pro with $16 \mathrm{~GB}$ of RAM running Mac OS X 10.6.8.

\subsection{List-Based Experiments}

Table 1 contains the results of our list experiments. For each program (leftmost column), we consider a randomly

\footnotetext{
${ }^{6}$ Hammer et al. 2014) report that for interactive, lazy usage patterns, ADAPTON substantially outperforms another state-of-the-art incremental technique, self-adjusting computation (SAC), which sometimes can incur significant slowdowns. We do not compare directly against SAC here.
}

\begin{tabular}{|c|r|l||r|r|r|}
\hline \multicolumn{5}{|c|}{ Batch-mode comparison ("demand all”) } \\
\hline Program & $\mathrm{n}$ & Edit & FS (ms) & $\mathrm{A}(\times)$ & NA (×) \\
\hline eager filter & $1 \mathrm{e} 4$ & insert & 21 & 0.178 & 1.29 \\
& & delete & 21 & 0.257 & 1.39 \\
& & replace & 21 & 0.108 & 1.27 \\
\hline eager map & $1 \mathrm{e} 4$ & insert & 21.6 & 0.0803 & 1.02 \\
& & delete & 21.6 & 0.0920 & 1.01 \\
& & replace & 21.6 & 0.0841 & 1.09 \\
\hline min & $1 \mathrm{e} 5$ & insert & 424 & 2790 & 2980 \\
& & delete & 424 & 4450 & 4720 \\
& & replace & 424 & 1850 & 2310 \\
\hline sum & $1 \mathrm{e} 5$ & insert & 421 & 785 & 833 \\
& & delete & 421 & 1140 & 1230 \\
& & replace & 421 & 727 & 733 \\
\hline reverse & $1 \mathrm{e} 5$ & insert & 197 & 0.0404 & 1.23 \\
& & delete & 197 & 0.764 & 1.19 \\
& & replace & 197 & 0.0404 & 1.23 \\
\hline median & $1 \mathrm{e} 4$ & insert & 3010 & 0.747 & 127 \\
& & delete & 3010 & 192 & 115 \\
& & replace & 3010 & 0.755 & 148 \\
\hline quickhull & $1 \mathrm{e} 4$ & insert & 853 & $0.0256^{*}$ & 3.78 \\
& & delete & 853 & $0.0270^{*}$ & 4.11 \\
& & replace & 853 & $0.0378^{*}$ & 3.86 \\
\hline mergesort & $1 \mathrm{e} 4$ & insert & 267 & 0.212 & 12.0 \\
& & delete & 267 & 11.0 & 10.1 \\
& & replace & 267 & 0.205 & 10.5 \\
\hline
\end{tabular}

(a) Speedups of batch-mode experiments

\begin{tabular}{|c|c|l||r||r|r|}
\hline \multicolumn{5}{|c|}{ Demand-driven comparison ("demand one") } \\
\hline Program & \multicolumn{1}{|c|}{$\mathrm{n}$} & Edit & FS (ms) & A $(\times)$ & NA $(\times)$ \\
\hline lazy filter & $1 \mathrm{e} 5$ & insert & 0.016 & 3.79 & 3.55 \\
& & delete & 0.016 & 18.1 & 16.3 \\
& & replace & 0.016 & 3.55 & 3.20 \\
\hline lazy map & $1 \mathrm{e} 5$ & insert & 0.016 & 4.08 & 3.79 \\
& & delete & 0.016 & 18.1 & 20.4 \\
& & replace & 0.016 & 3.71 & 3.62 \\
\hline reverse & $1 \mathrm{e} 5$ & insert & 188 & 0.067 & 2130 \\
& & delete & 188 & 50.8 & 4540 \\
& & replace & 188 & 0.068 & 2360 \\
\hline mergesort & $1 \mathrm{e} 4$ & insert & 63.4 & 96.3 & 369 \\
& & delete & 63.4 & 111 & 752 \\
& & replace & 63.4 & 86.2 & 336 \\
\hline quickhull & $1 \mathrm{e} 4$ & insert & 509 & $0.0628^{*}$ & 5.30 \\
& & delete & 509 & $0.0571^{*}$ & 5.52 \\
& & replace & 509 & $0.0856^{*}$ & 5.23 \\
\hline
\end{tabular}

(b) Speedups of demand-driven experiments

Table 1: List benchmarks

generated input of size $\mathrm{n}$ and three kinds of edits to it: insert, delete, and replace. For the first, we insert an element in the list; for the second, we delete the inserted element; for the last, we delete an element and then re-insert an element with a new value. Rather than consider only one edit position, we consider ten positions in the input list, spaced evenly $(1 / 10$ through the list, 2/10 through the list, etc.), and perform the edit at those positions, computing the average time across all ten edits. We report the median of seven trials of this experiment.

The table reports the time to perform recomputation from scratch, in milliseconds, in column FS, and then the speedup (or slow-down) factor compared to the from-scratch time 
for both ADAPTON, in column A, and NOMINAL ADAPTON, in column NA. Table 1a considers the case when all of the program's output is demanded, whereas Table $1 \mathrm{~b}$ considers the case when only one element of the output is demanded, thus measuring the benefits of both nominal and classic ADAPTON in a lazy setting. Note that in the lazy setting, FS sometimes also avoids complete recomputation, since thunks that are created but never forced are not executed.

Results: Demand All. Table 1a focuses on benchmarks where all of the output is demanded, or when there is only a single output value (sum and minimum). In these cases, several patterns emerge in the results.

First, for eager map (Section 2) and eager filter, NoMINAL ADAPTON gets modest speedup and breaks even, respectively, while ADAPTON gets slowdowns of one to two orders of magnitude. As Section 2 explains, ADAPTON recomputes and reallocates a linear number of output elements for each $\mathrm{O}(1)$ input change (insertion, deletion or replacement). By contrast, NOMINAL ADAPTON need not rebuild the prefix of the output lists.

Next, the benchmarks minimum and sum use the probabilistically balanced trees from Section 3.1 to do an incremental fold where, in expectation, only a logarithmic number of intermediate computations are affected by a small change. Due to this construction, both ADAPTON and NOMINAL ADAPTON get large speedups over from-scratch computation (up to $4720 \times$ ). NOMINAL ADAPTON tends to get slightly more speedup, since its use of names leads to less tree rebuilding. This is similar to, but not as asymptotically deep as, the eager map example $(O(\log n)$ here versus $O(n)$ above).

The next four benchmarks (reverse, mergesort, median, quickhull) show marked contrasts between the times for NOMINAL ADAPTON and ADAPTON: In all cases, NOMINAL ADAPTON gets a speedup (from about $4 \times$ to $148 \times$ ), whereas ADAPTON nearly always gets a slowdown. Two exceptions are the deletion changes that revert a prior insertion. In these cases, ADAPTON reuses the original cache information that it duplicates (at great expense) after the insertion. ADAPTON gets no speedup for quickhull, our most complex benchmark in this table. By contrast, NOMINAL ADAPTON performs updates orders of magnitude faster than ADAPTON and gets a speedup over from-scratch; the stars $(*)$ indicate that we ran quickhull at one tenth of the listed input size for ADAPTON, because otherwise it used too much memory due to having large memo tables but little reuse from them.

Results: Demand One. Table $1 \mathrm{~b}$ focuses on benchmarks where one (of many possible) outputs are demanded. In these cases, two patterns emerge. First, on simple lazy list benchmarks map and filter, ADAPTON and NOMINAL ADAPTON perform roughly the same, with ADAPTON getting slightly higher speedups than NOMINAL ADAPTON. These cases are good fits for ADAPTON's model, and names only add overhead.

\begin{tabular}{|c|c|l|r|r|r|}
\hline \multicolumn{6}{|c|}{ Batch-mode comparison ("demand all") } \\
\hline Program & $\mathrm{n}$ & Edit & FS $(\mathrm{ms})$ & $\mathrm{A}(\times)$ & NA $(\times)$ \\
\hline fact & $5 \mathrm{e} 3$ & rep1 & 945 & 0.520 & 10900 \\
& & swap1 & 947 & 2410 & 4740 \\
& & swap2 & 955 & 4740 & 6590 \\
& & ext & 847 & 0.464 & 0.926 \\
\hline intlog;fact & $2^{30}, 5 \mathrm{e} 3$ & swap & 849 & 0.413 & 3.18 \\
\hline array & 2 & rep11 & 191 & 0.323 & 6.52 \\
max & & rep12 & 191 & 0.310 & 7.62 \\
\hline matrix & $20 \times 20$ & swap1 & 4500 & 0.617 & 1.31 \\
mult & & swap2 & 4500 & 0.756 & 1.17 \\
& $25 \times 25$ & ext & 6100 & 1.50 & 1.55 \\
\hline
\end{tabular}

Table 2: Speedups of IMP interpreter experiments

Second, on more involved list benchmarks (reverse, mergesort and quickhull), NOMINAL ADAPTON delivers greater speedups (from $5 \times$ to $4540 \times$ ) than ADAPTON, which often delivers slowdowns. Two exceptions are mergesort, where ADAPTON delivers speedups, but is still up to $6.7 \times$ slower than NOMINAL ADAPTON, and the deletion changes, which — as in the table above-are fast because of spurious duplication in the insertion change.

In summary, NOMINAL ADAPTON consistently delivers speedups for small changes, while ADAPTON does so to a lesser extent, and much less reliably.

\subsection{Interpreter Experiments}

We tested the incremental behavior of the IMP interpreter with three basic forms of edits to the input programs: replacing values (replace), swapping subexpressions (swap), and increasing the size of the input (ext). These experiments all take the following form: evaluate an expression, mutate the expression, and then reevaluate.

- For fact, repl mutates the value of an unused variable; swapl reverses the order of two assignments at the start of the program; swap2 reverses the order of two assignments at the end; and ext increases the size of the input.

- For intlog;fact, swap swaps the two subprograms.

- For array max, repll replaces a value at the start of the array, and repl2 moves a value from the start to the end of the array.

- For matrix mult, swapl reverses the order of the initial assignments of the outer arrays of the input matrices; swap 2 reverses the order of the while loops that initialize the inner arrays of the input matrices; and ext extends the dimensions of the input arrays.

Results. Table 2 summarizes the results, presented the same way as the list benchmarks. We can see that NOMINAL ADAPTON provides a speedup over from-scratch computation in all but one case, and can provide dramatic speedups. In addition, NOMINAL ADAPTON consistently outperforms classic ADAPTON, in some cases providing a speedup where ADAPTON incurs a (sizeable) slowdown. 
The fact program's repl experiment shows significant performance improvement due to names. Classic ADAPTON dirties each intermediary environment and is forced to recompute. With the naming strategy outlined in Section 3.3 . the environment is identified without regard to the particular values inserted. Future computations that depend on the environment, but not the changed value in particular, are reused. The fact swap experiments show significant speedup for both classic ADAPTON and NOMINAL ADAPTON, because the trie map representation remains unchanged regardless of order of the assignments.

The remaining results fall into two categories. The edits made to intlog;fact, array max, and matrix mult's swaps show speedups between $1.17 \times$ and $7.62 \times$ with NOMINAL ADAPTON, while classic ADAPTON exhibits a slowdown, due to spending much of its time creating and evaluating new nodes in the DCG. NOMINAL ADAPTON, on the other hand, spends its time walking the already-present nodes and reusing many (from $25 \%$ to as much as $99 \%$ ) of them, with the added benefit of far better memory performance.

The last category includes the ext tests for fact (increasing the input value) and matrix mult (extending the dimensions of the input matrices). Such changes have pervasive effects on the rest of the computation and are a challenge to incremental reuse. Extension for matrix multiplication shows a modest speedup over the from-scratch running time for both nominal and classic ADAPTON. NOMINAL ADAPTON is able to reuse a third of the nodes created during the original run, while classic ADAPTON is not able to reuse any. Increasing the value of the input to factorial causes similar behavior, though the single, short loop prevents the amount of reuse from overcoming the from-scratch time.

\section{Related Work}

Here we survey past approaches to incremental computation, organizing our discussion into three categories: static approaches, dynamic approaches, and specialized approaches.

Static Approaches to IC. These approaches transform programs to derive a second program that can process "deltas"; the derived program takes as input the last (full) output and the representation of an input change, and produces (the representation of) the next output change. This program derivation is performed a priori, before any dynamic changes are issued. As such, static approaches have the advantage of not incurring dynamic space or time overhead, but also carry disadvantages that stem from not being dynamic in nature: They cannot handle programs with general recursion, and cannot take advantage of cached intermediate results, since by design, there are none (Cai et al. 2014; Liu and Teitelbaum 1995). Other static approaches transform programs into ones that cache and reuse past results, given a predefined class of input changes (Liu et al. 1998). Future work should explore an empirical comparison between these approaches and comtemporary dynamic approaches, described below.
Dynamic Approaches to IC. In contrast to static approaches, dynamic approaches attempt to trade space for time savings. A variety of dynamic approaches to IC have been proposed. Most early approaches fall into one of two camps: they either perform function caching of pure programs (Bellman 1957; McCarthy 1963; Michie 1968; Pugh 1988), or they support input mutation and employ some form of dynamic dependency graphs. However, the programming model advanced by earlier work on dependence graphs lacked features like general recursion and dynamic allocation, instead restricting programs to those expressible as attribute grammars (a language of declarative constraints over tree structures) (Demers et al. 1981; Reps 1982ab; Vogt et al. 1991).

Some recent general-purpose approaches to dynamic IC (SAC and ADAPTON) support general-purpose input structures and general recursion; internally, they use a notion of memoization to find and reuse portions of existing dependency graphs. As described in Hammer et al. (2014), SAC and ADAPTON differ greatly in the programming model they support (SAC is eager/batch-oriented whereas ADAPTON is demand-driven) and in how they represent dependency graphs. Consequently, they have different performance characteristics, with ADAPTON excelling at demand-driven and interactive settings, and SAC doing better in non-interactive, batch-oriented settings (Hammer et al.2014).

The presence of dynamic memory allocation in SAC poses a reuse problem due to "fresh" object identities, and thus benefits from a mechanism to deterministically match up identities from prior runs. Various past work on SAC addresses this problem in some form (Acar and Ley-Wild 2009: Acar et al. 2004, 2006, Hammer and Acar 2008) describing how to use "hints" or "keys." The reuse problem in NOMINAL ADAPTON is more general in nature than in $\mathrm{SAC}$, and thus requires a very different solution. For example, NOMINAL ADAPTON's DCG and more general memo tables do not impose SAC's total ordering of events, admitting more opportunities for reuse, but complicating the issue of assuring that names are not used more than once within a run. The use of thunks, which also need names, adds a further layer of complication. This paper addresses name reuse in this (more general) IC setting. Further, we address other naming issues, such as how to generate new names from existing ones (via fork) and how to determinize memo table creation (via named namespaces).

Ongoing research in programming languages and systems continues to generalize memoization. Bhatotia et al. (2015) extend memoization to parallel $\mathrm{C}$ and $\mathrm{C}++$ programs written against a traditional UNIX threading API. Bhatotia et al. (2011) extend memoization to distributed, cloudbased settings (MapReduce-style computations in particular). Chen et al. (2014) reduce the (often large) time and space overhead, which is pervasive in both SAC and in ADAPTON. In particular, they propose coarsening the granu- 
larity of dependence tracking, and report massive reductions (orders of magnitude) in space as a result. We believe that their approach ("probabilistic chunking") should be immediately applicable to our setting, as well as to classic ADAPTON. Indeed, early results for mergesort indicate up to an order-of-magnitude reduction in overhead.

Specialized Approaches to IC. Some recent approaches to IC are not general-purpose, but exploit domain-specific structure to handle input changes efficiently. DITTO incrementally checks invariants in Java programs, but is limited to invariant checking (Shankar and Bodík 2007). i3QL incrementally repairs database views (queries) when the underlying data changes due to insertions and removals of table rows (Mitschke et al. 2014).

Finally, reactive programming (especially functional reactive programming or FRP) shares some elements with incremental computation: both paradigms offer programming models for systems that strive to efficiently react to "outside changes"; internally, they use graph representations to model dependencies in a program that change over time (Cooper and Krishnamurthi 2006, Czaplicki and Chong 2013, Krishnaswami and Benton 2011). However, the chief aim of FRP is to provide a declarative means of specifying programs whose values are time-dependent (stored in signals), whereas the chief aim of IC is to provide time savings for small input changes (stored in special references). The different scope and programming model of FRP makes it hard to imagine using it to write an efficient incremental sorting algorithm, though it may be possible. On the other hand, IC would seem to be an appropriate mechanism for implementing an FRP engine, though the exact nature of this connection remains unclear.

\section{Conclusion}

This paper has presented nominal matching, a new strategy that general-purpose incremental computation can use to match a proposed computation against a prior, memoized one. With nominal matching, programmers may explicitly associate a name with a memoized computation, and matching is done by name equality. Nominal matching overcomes the conservativity of structural matching, the most commonly employed approach, which compares computations based on their structure and thus may fail to reuse prior results when it should (i.e., those that are not structurally identical but require little work to bring up-to-date). We have implemented nominal matching as part of NOMINAL ADAPTON, an extension to the ADAPTON general-purpose system for incremental computation, and endowed it with namespaces for more flexible management of names in practical programs. We have formalized NOMINAL ADAPTON's (and ADAPTON's) algorithms and proved them correct. We have implemented a variety of data structures and benchmark programs in NOMINAL ADAPTON. Performance experiments show that compared to ADAPTON (which employs structural matching) NOMINAL ADAPTON enjoys uniformly better performance, sometimes achieving many orders-ofmagnitude speedups over from-scratch computation when ADAPTON would suffer significant slowdowns.

\section{Acknowledgments}

We thank James Parker, Khoo Yit Phang, Robert Harper, as well as the anonymous reviewers of the program committees (including the AEC) for their questions, insights and feedback. We also thank James Parker for his contributions to an earlier prototype of the proposed design. This research was supported in part by NSF CCF-1116740, NSF CCF-1319666, DARPA FA8750-12-2-0106, and a gift from Mozilla Research.

\section{References}

U. A. Acar. Self-Adjusting Computation. PhD thesis, Department of Computer Science, Carnegie Mellon University, May 2005.

U. A. Acar and R. Ley-Wild. Self-adjusting computation with Delta ML. In Advanced Functional Programming. 2009.

U. A. Acar, G. E. Blelloch, and R. Harper. Adaptive memoization. Technical Report CMU-CS-03-208, Carnegie Mellon University, Nov. 2004.

U. A. Acar, G. E. Blelloch, M. Blume, R. Harper, and K. Tangwongsan. A library for self-adjusting computation. ENTCS, 148 (2), 2006.

J. Allen. Anatomy of LISP. McGraw-Hill, Inc., New York, NY, USA, 1978. ISBN 0-07-001115-X.

C. B. Barber, D. P. Dobkin, and H. Huhdanpaa. The quickhull algorithm for convex hulls. ACM Trans. Math. Softw., 22(4), Dec. 1996.

R. Bellman. Dynamic Programming. Princeton Univ. Press, 1957.

P. Bhatotia, A. Wieder, R. Rodrigues, U. A. Acar, and R. Pasquin. Incoop: MapReduce for incremental computations. In SOCC, 2011.

P. Bhatotia, P. Fonseca, U. A. Acar, B. B. Brandenburg, and R. Rodrigues. iThreads: A threading library for parallel incremental computation. In ASPLOS, 2015.

Y. Cai, P. G. Giarrusso, T. Rendel, and K. Ostermann. A theory of changes for higher-order languages: Incrementalizing $\lambda$-calculi by static differentiation. In PLDI, 2014.

Y. Chen, U. A. Acar, and K. Tangwongsan. Functional programming for dynamic and large data with self-adjusting computation. In ICFP, 2014.

G. H. Cooper and S. Krishnamurthi. Embedding dynamic dataflow in a call-by-value language. In ESOP, 2006.

E. Czaplicki and S. Chong. Asynchronous functional reactive programming for GUIs. In PLDI, 2013.

A. Demers, T. Reps, and T. Teitelbaum. Incremental evaluation of attribute grammars with application to syntax-directed editors. In POPL, 1981.

J.-C. Filliâtre and S. Conchon. Type-safe modular hash-consing. In Proceedings of the 2006 Workshop on ML, pages 12-19. ACM, 2006. 
M. Hammer and U. A. Acar. Memory management for selfadjusting computation. In ISMM, 2008.

M. Hammer, G. Neis, Y. Chen, and U. A. Acar. Self-adjusting stack machines. In ACM SIGPLAN Conference on ObjectOriented Programming, Systems, Languages, and Applications (OOPSLA), 2011.

M. A. Hammer, U. A. Acar, and Y. Chen. CEAL: a C-based language for self-adjusting computation. In ACM SIGPLAN Conference on Programming Language Design and Implementation, 2009.

M. A. Hammer, Y. P. Khoo, M. Hicks, and J. S. Foster. Adapton: Composable, demand-driven incremental computation. In PLDI, 2014.

M. A. Hammer, J. Dunfield, K. Headley, N. Labich, J. S. Foster, M. Hicks, and D. Van Horn. Incremental computation with names (extended version). arXiv:1503.07792 [cs.PL], 2015.

N. R. Krishnaswami and N. Benton. A semantic model for graphical user interfaces. In ICFP, 2011.

P. B. Levy. Call-by-push-value: A subsuming paradigm. In $T L C A$, 1999.

P. B. Levy. Call-By-Push-Value. PhD thesis, Queen Mary and Westfield College, University of London, 2001.

Y. A. Liu and T. Teitelbaum. Systematic derivation of incremental programs. Sci. Comput. Program., 24(1):1-39, 1995.
Y. A. Liu, S. Stoller, and T. Teitelbaum. Static caching for incremental computation. ACM Transactions on Programming Languages and Systems, 20(3):546-585, 1998.

J. McCarthy. A basis for a mathematical theory of computation. In Computer Programming and Formal Systems, 1963.

D. Michie. "Memo" functions and machine learning. Nature, 218: 19-22, 1968.

R. Mitschke, S. Erdweg, M. Köhler, M. Mezini, and G. Salvaneschi. i3QL: Language-integrated live data views. In $O O P$ SLA, 2014.

W. Pugh. Incremental Computation via Function Caching. PhD thesis, Cornell University, 1988.

W. Pugh and T. Teitelbaum. Incremental computation via function caching. In $P O P L, 1989$.

T. Reps. Generating Language-Based Environments. PhD thesis, Cornell University, Aug. 1982a.

T. Reps. Optimal-time incremental semantic analysis for syntaxdirected editors. In POPL, 1982b.

A. Shankar and R. Bodík. DITTO: Automatic incrementalization of data structure invariant checks (in Java). In PLDI, 2007.

H. Vogt, D. Swierstra, and M. Kuiper. Efficient incremental evaluation of higher order attribute grammars. In PLILP, 1991.

G. Winskel. The Formal Semantics of Programming Languages: An Introduction. MIT Press, 1993. 


\section{Supplement to "Incremental Computation with Names"}

This supplementary material contains additional details about how NOMINAL ADAPTON manages space, in Appendix A Our full from-scratch consistency result, along with the definitions and lemmas it uses, appears in Appendix B.

\section{A. Space management}

In a long-running program, memo tables could grow without bound. NOMINAL ADAPTON helps reduce table sizes, as we have already seen, but we still need a mechanism to clean out the tables when space becomes limited. A natural idea is to implement a memo table's mapping from name to DCG node with a weak reference, so that if the table is the only reference to the node, the node can be garbage collected when the system is short on space. This is not quite enough, though, because to implement dirtying, DCG edges are bidirectional. To avoid space leaks, it is critical to also make these backedges weak.

Unfortunately, using weak references for both memo tables and back edges (as implemented in the original ADAPTON) is generally unsound. ADAPTON supports an interactive pattern called swapping, wherein DCG components can be swapped in and out of the active DCG. Pathologically, during the time that a sub-computation is swapped out, the garbage collector could remove some of this DCG structure, but not all of it. In particular, it could null out some of its weak back edges, because these nodes are only reachable by weak references. But if this swapped out sub-computation is later swapped back into the DCG, these (weak) back edges will be gone, and we will potentially fail to dirty nodes that ought to be dirty, as future changes occur.

To fix the GC problem, we still use weak references for back edges, but use strong references for memo table entries, so that from the GC's point of view, all DCG nodes are always reachable. To implement safe space reclamation, we also implement reference counting of DCG nodes, where the counts reflect the number of strong edges reaching a node. When DCG edges are deleted, the reference counts of target nodes are decremented. Nodes that reach zero are not immediately collected; this allows thunks to be "resurrected" by the swapping pattern. Instead, we provide a flush operation for memo tables that deletes the strong mapping edge for all nodes with a count of zero, which means they are no longer reachable by the main program. Deletion is transitive: removing the node decrements the counts of nodes it points to, which may cause them to be deleted.

An interesting question is how to decide when to invoke flush; this is the system's eviction policy. One obvious choice is to flush when the system starts to run short of memory (based on a signal from the GC), which matches the intended effect of the unsound weak reference-based approach. But placing the eviction policy under the program's control opens other possibilities, e.g., the programmer could invoke flush when it is semantically clear that thunks cannot be restored. We leave to future work a further exploration of sensible eviction policies.

\section{B. Metatheory of $\lambda_{\text {NomA }}$}

\section{B.1 Overview}

Our main formal result in this paper, from-scratch consistency, states that given an evaluation derivation corresponding to an incremental computation, we can construct a derivation corresponding to a non-incremental computation that returns the same result and a corresponding graph. That is, the incremental computation is consistent with a computation in the simpler non-incremental system.

To properly state the consistency result, we need to define what it means for a graph to be well-formed (Section B.2), relate the incremental computation's DCG to the non-incremental computation's store (Section B.3), describe the sets of nodes read and written by an evaluation derivation (Section B.4), and prove a store weakening lemma (Section B.6). The consistency result itself is stated and proved in Section B.7.

\section{B.2 Graph Well-Formedness}

The judgment $\mathrm{G} \vdash \mathrm{H}$ wf is read " $\mathrm{H}$ is a well-formed subset of G". It implies that $\mathrm{H}$ is a linearization of a subset of G: within $\mathrm{H}$, information sources appear to the left of information sinks, dependency edges point to the left, and information flows to the right. Consequently, $\mathrm{H}$ is a dag.

For brevity, we write $\mathrm{G}$ wf for $\mathrm{G} \vdash \mathrm{G}$ wf, threading the entire graph $\mathrm{G}$ through the rules deriving $\mathrm{G} \vdash \mathrm{H}$ wf. The wellformedness rules in Figure 8 decompose the right-hand graph, and work as follows:

- For values (Grwf-val) and thunks with no cached result (Grwf-thunk), the rules only check the correspondence between $\mathrm{G}$ (the entire graph) and $\mathrm{H}$ (the subgraph).

- For thunks with a cached result, Grwf-thunkCache examines the outgoing edges. If they are all clean, then it checks that evaluating e again would not change the graph at all: $\mathcal{D}:: \mathrm{G} \vdash{ }_{\omega}^{p} e \Downarrow G$; t. If one or more edges are dirty, it checks that all incoming edges are dirty. 
$\mathrm{G} \vdash \mathrm{H}$ wf

$$
\begin{aligned}
& \overline{G \vdash \varepsilon w f} \text { Grwf-emp } \quad \frac{G \vdash H w f \quad G(p)=v}{G \vdash H, p: v w f} \text { Grwf-val } \quad \frac{G \vdash H w f \quad G(p)=e}{G \vdash H, p: e w f} \text { Grwf-thunk } \\
& \mathrm{G}(\mathrm{p})=(e, \mathrm{t}) \\
& \text { if all-clean-out }(G, p) \text { then }\left(\mathcal{D}:: G \vdash_{\omega}^{p} e \Downarrow G ; t\right) \\
& \frac{\mathrm{G} \vdash \mathrm{H} \text { wf } \quad \text { if not all-clean-out }(\mathrm{G}, \mathrm{p}) \text { then all-dirty-in }(\mathrm{G}, \mathrm{p})}{\mathrm{G} \vdash \mathrm{H}, \mathrm{p}:(e, \mathrm{t}) \mathrm{wf}} \text { Grwf-thunkCache } \\
& (p, a, \operatorname{dirty}, q) \in G \\
& q \in \operatorname{dom}(H) \\
& \frac{G \vdash H \text { wf } \quad \text { if }\left(p_{0}, a_{0}, b_{0}, p\right) \in G \text { then } b_{0}=\text { dirty }}{G \vdash H,(p, a, \text { dirty, } q) \text { wf }} \text { Grwf-dirtyEdge } \\
& (p, a, \text { clean }, q) \in G \\
& \text { consistent-action }(H, a, q) \\
& \frac{\mathrm{G} \vdash \mathrm{H} \text { wf } \quad \text { all-clean-out }(\mathrm{G}, \mathrm{q})}{\mathrm{G} \vdash \mathrm{H},(\mathrm{p}, \mathrm{a}, \text { clean, } \mathrm{q}) \mathrm{wf}} \text { Grwf-cleanEdge }
\end{aligned}
$$

Figure 8: Graph well-formedness rules

- Grwf-dirtyEdge checks that all edges flowing into a dirty edge are dirty: given edges from $p$ to $q$ and from $q$ to $r$, where the edge from $q$ to $r$ is dirty (meaning that $q$ depends on $r$ and $r$ needs to be recomputed), the edge from $p$ to $q$ should also be dirty. Otherwise, we would think we could reuse the result in $p$, even though $p$ (transitively) depends on $r$.

Conversely, if an edge from $p$ to $q$ is clean (Grwf-cleanEdge), then all edges out from $q$ must be clean (otherwise we would contradict the "transitive dirtiness" just described). Moreover, the action a stored in the edge from $p$ to $q$ must be consistent with the contents of $q$.

\section{B.3 From Graphs to Stores: the Restriction Function}

To relate the graph associated with an incremental evaluation to the store associated with a non-incremental evaluation, we define a function $\lfloor G\rfloor_{P}$ that restricts $G$ to a set of pointers $P$, drops cached thunk results $(*)$, and erases all edges $(* *)$ :

Definition B.1 (Restriction).

$$
\begin{array}{rlrl}
\lfloor\varepsilon\rfloor_{P} & =\varepsilon & & \\
\lfloor G, p: v\rfloor_{P} & =\lfloor G\rfloor_{P}, p: v & & \text { if } p \in P \\
\lfloor G, p: v\rfloor_{P} & =\lfloor G\rfloor_{P} & & \text { if } p \notin P \\
\lfloor G, p: e\rfloor_{P} & =\lfloor G\rfloor_{P}, p: e & & \text { if } p \in P \\
\lfloor G, p: e\rfloor_{P} & =\lfloor G\rfloor_{P} & & \text { if } p \notin P \\
(*) \quad\lfloor G, p:(e, t)\rfloor_{P} & =\lfloor G\rfloor_{P}, p: e & & \text { if } p \in P \\
\lfloor G, p:(e, t)\rfloor_{P} & =\lfloor G\rfloor_{P} & & \text { if } p \notin P \\
(* *) \quad\lfloor G,(p, a, b, q)\rfloor_{P} & =\lfloor G\rfloor_{P} &
\end{array}
$$

\section{B.4 Read and Write Sets}

Join and Merge Operations. To specify the read and write sets, we use a separating join $\mathrm{H}_{1} * \mathrm{H}_{2}$ on graphs: $\mathrm{H}_{1} * \mathrm{H}_{2}=$ $\left(\mathrm{H}_{1}, \mathrm{H}_{2}\right)$ if $\operatorname{dom}\left(\mathrm{H}_{1}\right) \perp \operatorname{dom}\left(\mathrm{H}_{2}\right)$, and undefined otherwise.

We also define a merge $\mathrm{H}_{1} \cup \mathrm{H}_{2}$ that is defined for subgraphs with overlapping domains, provided $\mathrm{H}_{1}$ and $\mathrm{H}_{2}$ are consistent with each other. That is, if $p \in \operatorname{dom}\left(H_{1}\right)$ and $p \in \operatorname{dom}\left(H_{2}\right)$, then $H_{1}(p)=H_{2}(p)$.

Definition B.2 (Reads/writes). The effect of an evaluation derived by $\mathcal{D}$, written $\mathcal{D}$ reads $\mathrm{R}$ writes $\mathrm{W}$, is defined in Figure 9 . 
$\mathcal{D}$ by Eval-term () reads $\varepsilon$ writes $\varepsilon$

$\mathcal{D}$ by Eval-app $\left(\mathcal{D}_{1}, \mathcal{D}_{2}\right)$ reads $R_{1} \cup\left(R_{2}-W_{1}\right)$ writes $W_{1} * W_{2}$ if $\mathcal{D}_{1}$ reads $R_{1}$ writes $W_{1}$ and $\mathcal{D}_{2}$ reads $R_{2}$ writes $W_{2}$

$\mathcal{D}$ by Eval-bind $\left(\mathcal{D}_{1}, \mathcal{D}_{2}\right)$ reads $R_{1} \cup\left(R_{2}-W_{1}\right)$ writes $W_{1} * W_{2}$ if $\mathcal{D}_{1}$ reads $R_{1}$ writes $W_{1}$ and $\mathcal{D}_{2}$ reads $R_{2}$ writes $W_{2}$

$\mathcal{D}$ by Eval-nest $\left(\mathcal{D}_{1}, \mathcal{D}_{2}\right)$ reads $R_{1} \cup\left(R_{2}-W_{1}\right)$ writes $W_{1} * W_{2}$ if $\mathcal{D}_{1}$ reads $R_{1}$ writes $W_{1}$ and $\mathcal{D}_{2}$ reads $R_{2}$ writes $W_{2}$

$\mathcal{D}$ by Eval-computeDep $\left(\mathcal{D}_{1}, \mathcal{D}_{2}\right)$ reads $R_{1} \cup R_{2}$ writes $W_{2}$ if $\mathcal{D}_{1}$ reads $R_{1}$ writes $W_{1}$ and $\mathcal{D}_{2}$ reads $R_{2}$ writes $W_{2}$ and $\operatorname{dom}\left(W_{1}\right) \subseteq \operatorname{dom}\left(W_{2}\right)$

\footnotetext{
$\mathcal{D}$ by $\operatorname{Eval}$-fix $\left(\mathcal{D}_{0}\right)$ reads $R$ writes $W$ if $\quad \mathcal{D}_{0}$ reads $R$ writes $W$

$\mathcal{D}$ by Eval-case $\left(\mathcal{D}_{0}\right)$ reads $R$ writes $W$ if $\quad \mathcal{D}_{0}$ reads $R$ writes $W$

$\mathcal{D}$ by Eval-split $\left(\mathcal{D}_{0}\right)$ reads $R$ writes $W$ if $\quad \mathcal{D}_{0}$ reads $R$ writes $W$

$\mathcal{D}$ by Eval-namespace $\left(\mathcal{D}_{0}\right)$ reads $R$ writes $W$ if $\quad \mathcal{D}_{0}$ reads $R$ writes $W$

$\mathcal{D}$ by Eval-fork() reads $\varepsilon$ writes $\varepsilon$

$\mathcal{D}$ by Eval-refDirty () reads $\varepsilon$ writes $q: v$ where $e=\operatorname{ref}(\mathbf{n m} k, v)$ and $q=k @ \omega$

$\mathcal{D}$ by Eval-refClean () reads $\varepsilon$ writes $q: v$ where $e=\operatorname{ref}(\mathbf{n m} k, v)$ and $q=k @ \omega$

$\mathcal{D}$ by Eval-thunkDirty () reads $\varepsilon$ writes $q: e_{0} \quad$ where $e=\operatorname{thunk}\left(\mathbf{n m} k, e_{0}\right)$

$\mathcal{D}$ by Eval-thunkClean() reads $\varepsilon$ writes $q: G(q)$ where $e=\operatorname{thunk}\left(\mathbf{n m} k, e_{0}\right)$

$\mathcal{D}$ by Eval-getClean () reads $q: \mathcal{v}$ writes $\varepsilon$ where $e=\operatorname{get}(\operatorname{ref} q)$ and $q=k @ \omega$ and $G(q)=v$

$\mathcal{D}$ by Eval-forceClean () reads $R^{\prime}, q:(e, t)$ writes $W^{\prime}$ where $e=$ force (thk $\left.q\right)$

and $\mathcal{D}^{\prime}$ reads $R^{\prime}$ writes $W^{\prime}$

where $\mathcal{D}^{\prime}$ is the derivation that computed $\mathrm{t}$ (see text)

$\mathcal{D}$ by Eval-scrubEdge $\left(\mathcal{D}_{0}\right)$ reads $R$ writes $W$ if $\quad \mathcal{D}_{0}$ reads $R$ writes $W$
}

Figure 9: Read- and write-sets of a derivation

This is a function over derivations. We write " $\mathcal{D}$ by $\mathcal{R}(\overrightarrow{\mathcal{D}})$ reads $\mathrm{R}$ writes $W$ " to mean that rule $\mathcal{R}$ concludes $\mathcal{D}$ and has subderivations $\overrightarrow{\mathcal{D}}$. For example, $\mathcal{D}$ by Eval-fix $\left(\mathcal{D}_{0}\right)$ reads $\mathrm{R}$ writes $\mathrm{W}$ provided that $\mathcal{D}_{0}$ reads $\mathrm{R}$ writes $W$ where $\mathcal{D}_{0}$ derives the only premise of Eval-fix.

In the Eval-forceClean case, we refer back to the derivation that (most recently) computed the thunk being forced (that is, the first subderivation of Eval-computeDep). A completely formal definition would take as input a mapping from pointers $q$ to sets $\mathrm{R}^{\prime}$ and $\mathrm{W}^{\prime}$, return this mapping as output, and modify the mapping in the Eval-computeDep case.

Agreement. We want to express a result (Lemma B.5 Respect for write-set) that evaluation only affects pointers in the write set $W$, leaving the contents of other pointers alone, so we define what it means to leave pointers alone:

Definition B.3 (Agreement on a pointer). Graphs $G_{1}$ and $G_{2}$ agree on $p$ iff either $\exp \left(G_{1}, p\right)=\exp \left(G_{2}, p\right)$, or $p: v \in G_{1}$ and $p: v \in \mathrm{G}_{2}$.

Definition B.4 (Agreement on a set of pointers). Graphs $G_{1}$ and $G_{2}$ agree on a set $P$ of pointers iff $G_{1}$ and $G_{2}$ agree on each $p \in P$.

Lemma B.5 (Respect for write-set).

If $\mathcal{D}:: \mathrm{G} \vdash \underset{\omega}{p} e \Downarrow G^{\prime} ; t$ where $\mathcal{D}$ is incremental and $\mathcal{D}$ reads $R$ writes $W$

then $\mathrm{G}^{\prime}$ agrees with $\mathrm{G}$ on $\operatorname{dom}(\mathrm{G})-\operatorname{dom}(\mathrm{W})$.

Proof. By a straightforward induction on $\mathcal{D}$, referring to Definition B.2

In the Eval-forceClean case, use the fact that $G^{\prime}$ differs from $G$ only in the addition of an edge, which does not affect agreement. 


\section{B.5 Satisfactory derivations}

In our main result (Theorem B.13), we will assume that all input and output graphs appearing within a derivation are wellformed, and that the read- and write-sets are defined:

Definition B.6 (Locally satisfactory).

A derivation $\mathcal{D}:: \mathrm{G}_{1} \vdash_{\omega}^{\mathrm{p}} \mathrm{e} \Downarrow \mathrm{G}_{2}$; $\mathrm{t}$ is locally satisfactory if and only if

(1) $G_{1}$ wf and $G_{2}$ wf

(2) $\mathcal{D}$ reads $R$ writes $W$ is defined

Definition B.7 (Globally satisfactory).

An evaluation derivation $\mathcal{D}:: \mathrm{H}_{1} \vdash_{\omega}^{p}$ e $\Downarrow \mathrm{H}_{2} ; \mathrm{t}$ is globally satisfactory, written $\mathcal{D}$ satisfactory, if and only if $\mathcal{D}$ is locally satisfactory and all its subderivations are locally satisfactory.

\section{B.6 Weakening}

The main result needs to construct a non-incremental derivation in a different order from the given incremental derivation. In particular, the first evaluation $\mathcal{D}_{1}$ done in Eval-computeDep will be done "later" in the non-incremental derivation. Since it is done later, the graph may have new material $\mathrm{G}^{\prime}$, and we need a weakening lemma to move from a non-incremental evaluation of $\mathcal{D}_{1}$ (obtained through the induction hypothesis) to a non-incremental evaluation over the larger graph.

Lemma B.8 (Weakening (non-incremental)).

If $\mathcal{D}:: \mathrm{G}_{1} \vdash_{\omega}^{\mathrm{p}} \mathrm{e} \Downarrow \mathrm{G}_{2} ; \mathrm{t}$ and $\mathcal{D}$ is non-incremental

and $\mathrm{G}^{\prime}$ is disjoint from $\mathrm{G}_{1}$ and $\mathrm{G}_{2}$

then $\mathcal{D}^{\prime}:: \mathrm{G}_{1}, \mathrm{G}^{\prime} \vdash_{\omega}^{\mathrm{p}} \mathrm{e} \Downarrow \mathrm{G}_{2}, \mathrm{G}^{\prime} ; \mathrm{t}$ where $\mathcal{D}^{\prime}$ is non-incremental.

Proof. By induction on $\mathcal{D}$.

- Cases Eval-term, Eval-app, Eval-fix, Eval-bind, Eval-case, Eval-split, Eval-fork, Eval-namespace, Eval-nest:

These rules do not manipulate the graph, so just use the i.h. on each subderivation, then apply the same rule.

- Case Eval-refPlain: Since $\mathrm{G}_{1}\{q \mapsto v\}=\mathrm{G}_{2}$, we have $\mathrm{q} \in \operatorname{dom}\left(\mathrm{G}_{2}\right)$.

It is given that $\operatorname{dom}\left(G^{\prime}\right) \perp \operatorname{dom}\left(G_{2}\right)$. Therefore $q \notin \operatorname{dom}\left(G_{1}, G^{\prime}\right)$.

By definition, $\left(\mathrm{G}_{1}, \mathrm{G}^{\prime}\right)\{\mathrm{q} \mapsto v\}=\left(\mathrm{G}_{2}, \mathrm{G}^{\prime}\right)$.

Apply Eval-refPlain.

- Case Eval-thunkPlain: Similar to the Eval-refPlain case.

- Case Eval-forcePlain: We have $\exp \left(G_{1}, q\right)=e_{0}$. Therefore $\exp \left(G_{1}, G^{\prime}, q\right)=e_{0}$. Use the i.h. and apply Eval-forcePlain.

- Case Eval-getPlain: Similar to the Eval-forcePlain case.

\section{B.7 Main result: From-scratch consistency}

At the highest level, the main result (Theorem B.13) says:

\section{First approximation}

If $\mathrm{H}_{1} \vdash_{\omega}^{p} e \Downarrow \mathrm{H}_{2}$; t by an incremental derivation, then $H_{1}^{\prime} \vdash_{\omega}^{p} e \Downarrow H_{2}^{\prime} ; t$, where $H_{1}^{\prime}$ is a non-incremental version of $H_{1}$ and $H_{2}^{\prime}$ is a non-incremental version of $H_{2}$.

Using the restriction function from Section B.3, we can refine this statement:

\section{Second approximation}

If $\mathrm{H}_{1} \vdash_{\omega}^{\mathrm{p}} \mathrm{e} \Downarrow \mathrm{H}_{2}$; t by an incremental derivation and $\mathrm{P}_{1} \subseteq \operatorname{dom}\left(\mathrm{H}_{1}\right)$, then $\left\lfloor\mathrm{H}_{1}\right\rfloor_{\mathrm{P}_{1}} \vdash_{\omega}^{\mathrm{p}} \mathrm{e} \Downarrow\left\lfloor\mathrm{H}_{2}\right\rfloor_{\mathrm{P}_{2}} ; \mathrm{t}$ by a non-incremental derivation, for some $\mathrm{P}_{2}$ such that $\mathrm{P}_{1} \subseteq \mathrm{P}_{2}$.

Here, the pointer set $P_{1}$ gives the scope of the non-incremental input graph $\left\lfloor H_{1}\right\rfloor P_{1}$, and we construct $P_{2}$ describing the non-incremental output graph $\left\lfloor\mathrm{H}_{2}\right\rfloor \mathrm{P}_{2}$.

We further refine this statement by involving the derivation's read- and write-sets: the read set $R$ must be contained in $P_{1}$, the write set $W$ must be disjoint from $P_{1}$ (written $\operatorname{dom}(W) \perp P_{1}$ ), and $P_{2}$ must be exactly $P_{1}$ plus $W$. In the non-incremental semantics, the store should grow monotonically, so we will also show $\left\lfloor\mathrm{H}_{1}\right\rfloor \mathrm{P}_{1} \subseteq\left\lfloor\mathrm{H}_{2}\right\rfloor_{\mathrm{P}_{2}}$ :

\section{Third approximation}

If $\mathrm{H}_{1} \vdash_{\omega}^{\mathrm{p}} \mathrm{e} \Downarrow \mathrm{H}_{2}$; $\mathrm{t}$ by an incremental derivation $\mathcal{D}$ with $\mathcal{D}$ reads $\mathrm{R}$ writes $W$, and $\mathrm{P}_{1} \subseteq \operatorname{dom}\left(\mathrm{H}_{1}\right)$ such that $\operatorname{dom}(\mathrm{R}) \subseteq \mathrm{P}_{1}$ and $\operatorname{dom}(\mathrm{W}) \perp \mathrm{P}_{1}$ 
then $\left\lfloor\mathrm{H}_{1}\right\rfloor \mathrm{P}_{1} \vdash_{\omega}^{\mathrm{p}} \mathrm{e} \Downarrow\left\lfloor\mathrm{H}_{2}\right\rfloor \mathrm{P}_{2} ; \mathrm{t}$

where $\left\lfloor\mathrm{H}_{1}\right\rfloor \mathrm{P}_{1} \subseteq\left\lfloor\mathrm{H}_{2}\right\rfloor \mathrm{P}_{2}$ and $\mathrm{P}_{2}=\mathrm{P}_{1} \cup \operatorname{dom}(W)$.

Even this refinement is not quite enough, because the incremental system can perform computations in a different order than the non-incremental system. Specifically, the Eval-computeDep rule carries out a subcomputation first, then continues with a larger computation that depends on the subcomputation. The subcomputation does not fit into the non-incremental derivation at that point; non-incrementally, the subcomputation is performed when it is demanded by the larger computation. Thus, we can't just apply the induction hypothesis on the subcomputation.

However, the subcomputation is "saved" in its (incremental) output graph. So we incorporate an invariant that all thunks with cached results in the graph "are consistent", that is, they satisfy a property similar to the overall consistency result. When this is the case, we say that the graph is from-scratch consistent. The main result, then, will assume that the input graph is fromscratch consistent, and show that the output graph remains from-scratch consistent. Since the graph can grow in the interval between the subcomputation of Eval-computeDep and the point where the subcomputation is demanded, we require that the output graph $\mathrm{G}_{2}$ of the saved derivation does not contradict the larger, newer graph $\mathrm{H}$. This is part (4) in the next definition. (We number the parts from (i) to (ii) and then from (1) so that they mostly match similar parts in the main result.)

Definition B.9 (From-scratch consistency of a derivation).

A derivation $\mathcal{D}_{i}:: \mathrm{G}_{1} \vdash_{\omega}^{\mathrm{p}} \mathrm{e} \Downarrow \mathrm{G}_{2} ; \mathrm{t}$ is

from-scratch consistent for $\mathrm{P}_{\mathrm{q}} \subseteq \operatorname{dom}\left(\mathrm{G}_{1}\right)$ up to $\mathrm{H}$ if and only if

(i) $\mathcal{D}_{i}$ satisfactory where $\mathcal{D}_{i}$ reads $\mathrm{R}$ writes $\mathrm{W}$

(ii) $\operatorname{dom}(\mathrm{R}) \subseteq \mathrm{P}_{\mathrm{q}}$ and $\operatorname{dom}(\mathrm{W}) \perp \mathrm{P}_{\mathrm{q}}$

(1) there exists a non-incremental derivation $\mathcal{D}_{\mathrm{ni}}::\left\lfloor\mathrm{G}_{1}\right\rfloor \mathrm{P}_{\mathrm{q}} \vdash_{\mathrm{\omega}}^{\mathrm{p}} \mathrm{e} \Downarrow\left\lfloor\mathrm{G}_{2}\right\rfloor \mathrm{P}_{2} ; \mathrm{t}$

(2) $\left\lfloor\mathrm{G}_{1}\right\rfloor \mathrm{P}_{\mathrm{q}} \subseteq\left\lfloor\mathrm{G}_{2}\right\rfloor \mathrm{P}_{2}$

(3) $\mathrm{P}_{2}=\mathrm{P}_{\mathrm{q}} \cup \operatorname{dom}(\mathrm{W})$

(4) $\left\lfloor\mathrm{G}_{2}\right\rfloor_{\mathrm{P}_{2}} \subseteq\lfloor\mathrm{H}\rfloor_{\operatorname{dom}(\mathrm{H})}$

Definition B.10 (From-scratch consistency of graphs).

A graph $\mathrm{H}$ is from-scratch consistent

if, for all $\mathrm{q} \in \operatorname{dom}(\mathrm{H})$ such that $\mathrm{H}(\mathrm{q})=(\mathrm{e}, \mathrm{t})$,

there exists $\mathcal{D}_{\mathrm{q}}:: \mathrm{G}_{1} \vdash_{\mathrm{w}}^{\mathrm{p}} \mathrm{e} \Downarrow \mathrm{G}_{2}$; $\mathrm{t}$

and $\mathrm{P}_{\mathrm{q}} \subseteq \operatorname{dom}\left(\mathrm{G}_{1}\right)$

such that $\mathcal{D}_{\mathrm{q}}$ is from-scratch consistent (Definition B.9) for $\mathrm{P}_{\mathrm{q}}$ up to $\mathrm{H}$.

The proof of the main result must maintain that the graph is from-scratch consistent as the graph becomes larger, for which Lemma B.12 Consistent graph extension is useful.

Lemma B.11 (Consistent extension).

If $\mathcal{D}_{\mathrm{q}}$ is from-scratch consistent for $\mathrm{P}_{\mathrm{q}}$ up to $\mathrm{H}$

and $\mathrm{H} \subseteq \mathrm{H}^{\prime}$

then $\mathcal{D}_{\mathrm{q}}$ is from-scratch consistent for $\mathrm{P}_{\mathrm{q}}$ up to $\mathrm{H}^{\prime}$.

Proof. Only part (4) of Definition B.9 involves the "up to" part of from-scratch consistency, so we already have (i)-(iii) and (1)-(3).

We have (4) $\left\lfloor\mathrm{G}_{2}\right\rfloor_{\mathrm{P}_{2}} \subseteq\lfloor\mathrm{H}\rfloor_{\operatorname{dom}(\mathrm{H})}$. Using our assumptions, $\lfloor\mathrm{H}\rfloor_{\operatorname{dom}(\mathrm{H})} \subseteq\left\lfloor\mathrm{H}^{\prime}\right\rfloor_{\operatorname{dom}\left(\mathrm{H}^{\prime}\right)}$.

Therefore (4) $\left\lfloor\mathrm{G}_{2}\right\rfloor_{\mathrm{P}_{2}} \subseteq\left\lfloor\mathrm{H}^{\prime}\right\rfloor_{\operatorname{dom}\left(\mathrm{H}^{\prime}\right)}$.

Lemma B.12 (Consistent graph extension).

If $\mathrm{H}$ is from-scratch consistent

and $\mathrm{H} \subseteq \mathrm{H}^{\prime}$

and, for all $\mathrm{q} \in \operatorname{dom}\left(\mathrm{H}^{\prime}\right)-\operatorname{dom}(\mathrm{H})$ such that $\mathrm{H}^{\prime}(\mathrm{q})=(\mathrm{e}, \mathrm{t})$,

there exists $\mathcal{D}_{\mathrm{q}}:: \mathrm{G}_{1} \vdash_{\mathrm{w}}^{\mathrm{p}} \mathrm{e} \Downarrow \mathrm{G}_{2} ; \mathrm{t}$ and $\mathrm{P}_{\mathrm{q}} \subseteq \operatorname{dom}\left(\mathrm{G}_{1}\right)$

such that $\mathcal{D}_{\mathrm{q}}$ is from-scratch consistent (Definition B.9)

for $\mathrm{P}_{\mathrm{q}}$ up to $\mathrm{H}^{\prime}$,

then $\mathrm{H}^{\prime}$ is from-scratch consistent.

Proof. Use Lemma B.11 Consistent extension on each "old" pointer with a cached result in $\mathrm{H}$, then apply the definitions for each "new" pointer with a cached result in $\operatorname{dom}\left(\mathrm{H}^{\prime}\right)-\operatorname{dom}(\mathrm{H})$. 
At last, we can state and prove the main result, which corresponds to the "third approximation" above, plus the invariant that the graph is from-scratch consistent (parts (iii) and (4)).

We present most of the proof in a line-by-line style, with the judgment or proposition being derived in the left column, and its justification in the right column. In each case, we need to show four different things (1)-(4), some of which are obtained midway through the case, so we highlight these with "(1) ", and so on.

Theorem B.13 (From-scratch consistency).

Given an incremental $\mathcal{D}_{i}:: \mathrm{H}_{1} \vdash_{\omega}^{p}$ e $\Downarrow \mathrm{H}_{2}$; t where

(i) $\mathcal{D}_{i}$ satisfactory where $\mathcal{D}$ reads $R$ writes $W$

(ii) a set of pointers $\mathrm{P}_{1} \subseteq \operatorname{dom}\left(\mathrm{H}_{1}\right)$ is such that $\operatorname{dom}(\mathrm{R}) \subseteq \mathrm{P}_{1}$ and $\operatorname{dom}(\mathrm{W}) \perp \mathrm{P}_{1}$

(iii) $\mathrm{H}_{1}$ is from-scratch consistent (Definition B.10)

\section{then}

(1) there exists a non-incremental $\mathcal{D}_{\mathrm{ni}}::\left\lfloor\mathrm{H}_{1}\right\rfloor_{\mathrm{P}_{1}} \vdash_{\omega}^{\mathrm{p}} \mathrm{e} \Downarrow\left\lfloor\mathrm{H}_{2}\right\rfloor_{\mathrm{P}_{2}} ; \mathrm{t}$

(2) $\left\lfloor\mathrm{H}_{1}\right\rfloor_{\mathrm{P}_{1}} \subseteq\left\lfloor\mathrm{H}_{2}\right\rfloor \mathrm{P}_{2}$

(3) $\mathrm{P}_{2}=\mathrm{P}_{1} \cup \operatorname{dom}(W)$

(4) $\mathrm{H}_{2}$ is from-scratch consistent (Definition B.10).

Proof. By induction on $\mathcal{D}_{i}:: \mathrm{H}_{1} \vdash_{\omega}^{p}$ e $\Downarrow \mathrm{H}_{2} ; \mathrm{t}$.

- Case Eval-term: By Definition B.2. $\mathrm{W}=\varepsilon$, so let $\mathrm{P}_{2}=\mathrm{P}_{1}$.

(1) Apply Eval-term.

(2) We have $\mathrm{H}_{1}=\mathrm{H}_{2}$ and $\mathrm{P}_{1}=\mathrm{P}_{2}$ so $\left\lfloor\mathrm{H}_{1}\right\rfloor \mathrm{P}_{1}=\left\lfloor\mathrm{H}_{2}\right\rfloor \mathrm{P}_{2}$.

(3) It follows from $\operatorname{dom}(W)=\emptyset$ and $P_{2}=P_{1}$ that $P_{2}=P_{1} \cup \operatorname{dom}(W)$.

(4) It is given (iii) that $\mathrm{H}_{1}$ is from-scratch consistent.

We have $\mathrm{H}_{1}=\mathrm{H}_{2}$, so $\mathrm{H}_{2}$ is from-scratch consistent.

- Case Eval-fork: Similar to the Eval-term case.

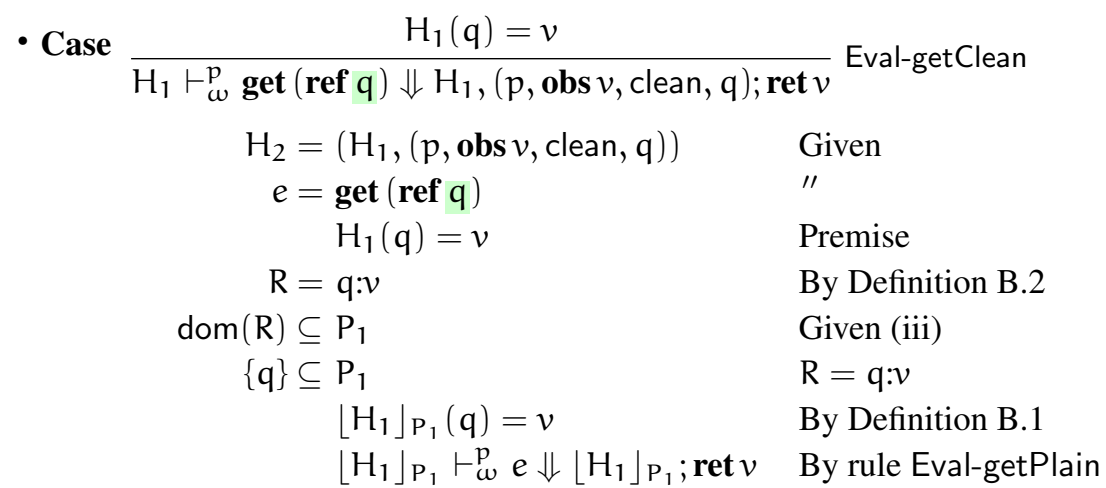

Let $P_{2}$ be $P_{1}$.
(1) $\leftrightarrow$ $\left\lfloor\mathrm{H}_{1}\right\rfloor_{\mathrm{P}_{1}}=\left\lfloor\mathrm{H}_{2}\right\rfloor \mathrm{P}_{1}$
By def. of restriction
$\left\lfloor\mathrm{H}_{1}\right\rfloor \mathrm{P}_{1} \vdash_{\omega}^{\mathrm{p}} \mathrm{e} \Downarrow\left\lfloor\mathrm{H}_{2}\right\rfloor \mathrm{P}_{1} ;$ ret $v$
By above equality
$W=\varepsilon$
By Definition B.2
(2) $\left\lfloor\mathrm{H}_{1}\right\rfloor \mathrm{P}_{1} \subseteq\left\lfloor\mathrm{H}_{2}\right\rfloor \mathrm{P}_{1}$
(3)
$\mathrm{P}_{2}=\mathrm{P}_{1} \cup \operatorname{dom}(\varepsilon)$
If $=$ then $\subseteq$
$\mathrm{H}_{1}$ from-scratch consistent
$\mathrm{P}_{2}=\mathrm{P}_{1}$
(4)
$\mathrm{H}_{2}$ from-scratch consistent
Given (iii)
$\mathrm{H}_{2}$ differs from $\mathrm{H}_{1}$ only in its edges

- Case $\frac{q=k @ \omega \quad H_{1}\left\{q \mapsto e_{0}\right\}=G_{2} \quad \operatorname{dirty-paths-in}\left(G_{2}, q\right)=G_{3}}{H_{1} \vdash_{\omega}^{p} \text { thunk }\left(\operatorname{nm} k, e_{0}\right) \Downarrow G_{3},\left(p, \text { alloc } e_{0}, \text { clean, } q\right) ; \text { ret }(\text { thk } q)}$ Eval-thunkDirty 


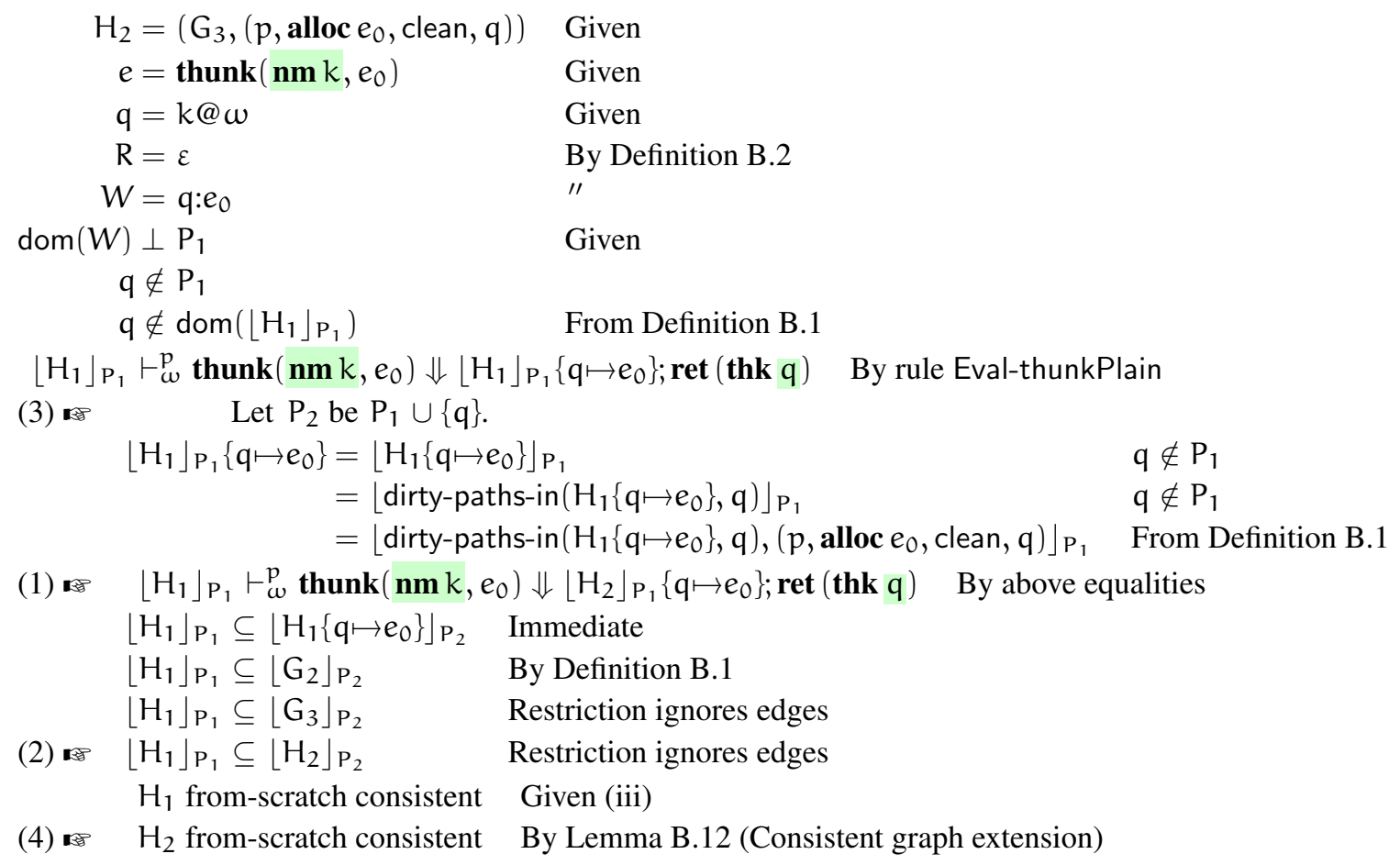

- Case $\quad \mathrm{q}=\mathrm{k} @ \omega \quad \exp \left(\mathrm{H}_{1}, \mathrm{q}\right)=e_{0}$

$\mathrm{H}_{1} \vdash_{\omega}^{\mathrm{p}} \operatorname{thunk}\left(\mathbf{n m k}, e_{0}\right) \Downarrow \mathrm{H}_{1},\left(p, \operatorname{alloc} e_{0}\right.$, clean, $\left.q\right) ; \operatorname{ret}($ thk $q)$ Eval-thunkClean

$$
\begin{aligned}
\mathrm{H}_{2} & =\left(\mathrm{H}_{1},\left(p, \text { alloc } e_{0}, \text { clean }, q\right)\right) & & \text { Given } \\
\mathrm{e} & =\operatorname{thunk}\left(\mathbf{n m} k, e_{0}\right) & & \text { Given } \\
\mathrm{q} & =k @ \omega & & \text { Given } \\
W & =\mathrm{q}: e_{0} & & \text { By Definition B.2 } \\
\operatorname{dom}(W) & \perp \mathrm{P}_{1} & & \text { Given }
\end{aligned}
$$$$
\mathrm{q} \notin \mathrm{P}_{1}
$$$$
\mathrm{q} \notin \operatorname{dom}\left(\left\lfloor\mathrm{H}_{1}\right\rfloor_{\mathrm{P}_{1}}\right) \quad \text { From Definition B.1 }
$$

$\left\lfloor\mathrm{H}_{1}\right\rfloor_{\mathrm{P}_{1}} \vdash_{\omega}^{\mathrm{p}}$ thunk $\left(\mathbf{n m} k, e_{0}\right) \Downarrow\left\lfloor\mathrm{H}_{1}\right\rfloor_{\mathrm{P}_{1}}\left\{\mathbf{q} \mapsto e_{0}\right\}$; ret (thk q) By rule Eval-thunkPlain $\left\lfloor H_{1}\right\rfloor_{\mathrm{P}_{1}}\left\{q \mapsto e_{0}\right\}=\left\lfloor\mathrm{H}_{1},\left(\mathrm{p} \text {, alloc } e_{0} \text {, clean, } q\right)\right\rfloor_{\mathrm{P}_{1}} \quad$ From Definition B.1 and $\exp \left(\mathrm{H}_{1}, \mathrm{q}\right)=e_{0}$ (1) $\left\lfloor\mathrm{H}_{1}\right\rfloor_{\mathrm{P}_{1}} \vdash_{\omega}^{\mathrm{p}} \operatorname{thunk}\left(\mathbf{n m} \mathrm{k}, e_{0}\right) \Downarrow\left\lfloor\mathrm{H}_{2}\right\rfloor_{\mathrm{P}_{1}}\left\{\mathrm{q} \mapsto e_{0}\right\} ;$ ret (thk q) By above equalities Since $H_{2}=H_{1}$, (p, alloc $e_{0}$, clean, $\left.q\right)$, parts (2)-(4) are straightforward.

- Case Eval-refDirty: Similar to the Eval-thunkDirty case.

- Case Eval-refClean: Similar to the Eval-thunkClean case.

- Case $\frac{\mathrm{H}_{1} \vdash_{\omega}^{\mathrm{p}} e_{1} \Downarrow \mathrm{H}^{\prime} ; \text { ret } v \quad \mathrm{H}^{\prime} \vdash_{w}^{\mathrm{p}}[v / x] e_{2} \Downarrow \mathrm{H}_{2} ; \mathrm{t}}{\mathrm{H}_{1} \vdash_{\omega}^{\mathrm{p}} \text { let } x \leftarrow e_{1} \text { in } e_{2} \Downarrow \mathrm{H}_{2} ; \mathrm{t}}$ Eval-bind

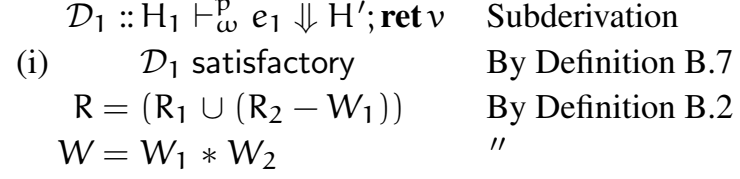

$$
\begin{array}{rll}
\operatorname{dom}\left(R_{1} \cup\left(R_{2}-W_{1}\right)\right) \subseteq & P_{1} & \text { Given } \\
\operatorname{dom}\left(W_{1} * W_{2}\right) \perp & P_{1} & \text { Given } \\
& \mathcal{D}_{1} \text { reads } R_{1} \text { writes } W_{1} & " \\
& \mathcal{D}_{2} \text { reads } R_{2} \text { writes } W_{2} & \prime
\end{array}
$$


(ii) $\operatorname{dom}\left(\mathrm{R}_{1}\right) \subseteq \mathrm{P}_{1}$

(ii) $\operatorname{dom}\left(\mathrm{W}_{2}\right) \perp \mathrm{P}_{1}$

$\left\lfloor\mathrm{H}_{1}\right\rfloor \mathrm{P}_{1} \subseteq\left\lfloor\mathrm{H}^{\prime}\right\rfloor_{\mathrm{P}^{\prime}}$

$$
\mathrm{P}^{\prime}=\mathrm{P}_{1} \cup \operatorname{dom}\left(\mathrm{W}_{1}\right)
$$

$\mathrm{H}^{\prime}$ from-scratch consistent

(i)

$$
\mathcal{D}_{2}:: \mathrm{H}^{\prime} \vdash_{\omega}^{\mathrm{p}}[v / \mathrm{x}] \mathrm{e}_{2} \Downarrow \mathrm{H}_{2} ; \mathrm{t}
$$

$\mathcal{D}_{2}$ satisfactory

$\operatorname{dom}\left(R_{1} \cup\left(R_{2}-W_{1}\right)\right) \subseteq P_{1}$

$\operatorname{dom}\left(R_{2}-W_{1}\right) \subseteq P_{1}$

$\operatorname{dom}\left(R_{2}\right) \subseteq P_{1} \cup \operatorname{dom}\left(W_{1}\right)$

(ii)

$$
\operatorname{dom}\left(R_{2}\right) \subseteq P^{\prime}
$$

$\operatorname{dom}\left(\mathrm{W}_{1} * \mathrm{~W}_{2}\right) \perp \mathrm{P}_{1}$

$\operatorname{dom}\left(W_{1}\right) \perp \operatorname{dom}\left(W_{2}\right)$

$\operatorname{dom}\left(W_{2}\right) \perp \mathrm{P}_{1} \cup \operatorname{dom}\left(W_{1}\right)$

(ii)

$$
\operatorname{dom}\left(W_{2}\right) \perp \mathrm{P}^{\prime} \quad \mathrm{P}^{\prime}=\mathrm{P}_{1} \cup \operatorname{dom}\left(\mathrm{W}_{1}\right)
$$$$
\left\lfloor\mathrm{H}^{\prime}\right\rfloor \mathrm{P}^{\prime} \subseteq\left\lfloor\mathrm{H}_{2}\right\rfloor \mathrm{P}_{2}
$$$$
\mathrm{P}_{2}=\mathrm{P}^{\prime} \cup \operatorname{dom}\left(\mathrm{W}_{2}\right)
$$

(4) $\mathrm{H}_{2}$ from-scratch consistent

(2) $\left\lfloor\mathrm{H}_{1}\right\rfloor_{\mathrm{P}_{1}} \subseteq\left\lfloor\mathrm{H}_{2}\right\rfloor_{\mathrm{P}_{2}}$

$$
P_{2}=P_{1} \cup \operatorname{dom}\left(W_{1}\right) \cup \operatorname{dom}\left(W_{2}\right)
$$

(3)

$$
\mathrm{P}_{2}=\mathrm{P}_{1} \cup \operatorname{dom}(W)
$$

$$
\begin{aligned}
& \left\lfloor\mathrm{H}_{1}\right\rfloor_{\mathrm{P}_{1}} \vdash_{\omega}^{\mathrm{p}} e_{1} \Downarrow\left\lfloor\mathrm{H}^{\prime}\right\rfloor_{\mathrm{P}^{\prime}} ; \text { ret } v \\
& \left\lfloor\mathrm{H}^{\prime}\right\rfloor_{\mathrm{P}^{\prime}} \vdash_{\omega}^{\mathrm{p}}[v / x] e_{2} \Downarrow\left\lfloor\mathrm{H}_{2}\right\rfloor_{\mathrm{P}_{2}} ; t
\end{aligned}
$$

$\operatorname{dom}\left(R_{1} \cup\left(R_{2}-W_{1}\right)\right) \subseteq P_{1}$

$\operatorname{dom}\left(W_{1} * W_{2}\right) \perp P_{1}$

By i.h.

II

II

"I

Subderivation

By Definition B.7

Given

$\mathrm{P}^{\prime}=\mathrm{P}_{1} \cup \operatorname{dom}\left(\mathrm{W}_{1}\right)$

(1) $\left\lfloor\mathrm{H}_{1}\right\rfloor_{\mathrm{P}_{1}} \vdash_{\omega}^{\mathrm{p}}$ let $x \leftarrow e_{1}$ in $e_{2} \Downarrow\left\lfloor\mathrm{H}_{2}\right\rfloor_{\mathrm{P}_{2}} ; t \quad$ By rule Eval-bind

- Case Eval-app: Similar to the Eval-bind case.

- Case Eval-nest: Similar to the Eval-bind case.

- Case Eval-fix: The input and output graphs of the subderivation match those of the conclusion, as do the read and write sets according to Definition B.2 Thus, we can just use the i.h. and apply Eval-fix.

- Case Eval-case: Similar to the Eval-fix case.

- Case Eval-split: Similar to the Eval-case case.

- Case Eval-namespace: Similar to the Eval-fix case.

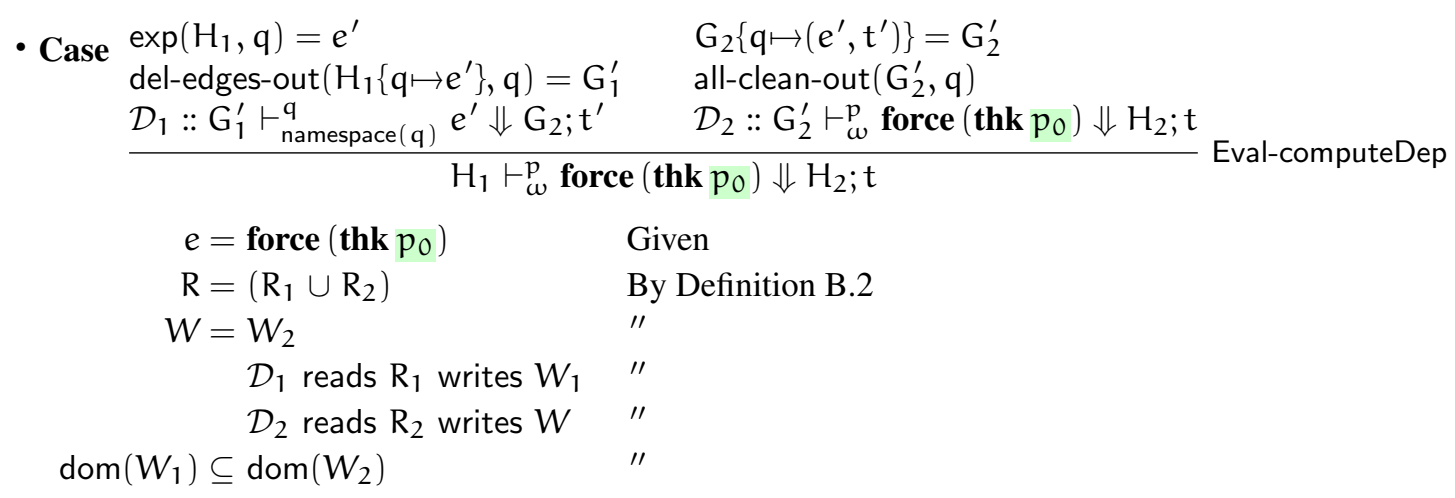

We don't immediately need to apply the i.h. to $\mathcal{D}_{1}$, because that computation will be done later in the reference derivation. But we do need to apply the i.h. to $\mathcal{D}_{2}:: \mathrm{G}_{2}^{\prime} \vdash_{\omega}^{\mathrm{p}} \mathrm{e} \Downarrow \mathrm{H}_{2}$; t. So we need to show part (iii) of the statement, which says that each cached computation in the input graph is consistent with respect to an earlier version of the graph. 
Since we're adding such a computation $e^{\prime}$ in $G_{2}^{\prime}$, which is the input graph of $\mathcal{D}_{2}$, we have to show that the computation of $e^{\prime}$ (by $\mathcal{D}_{1}$ ) is consistent, which means applying the i.h. to $\mathcal{D}_{1}$.

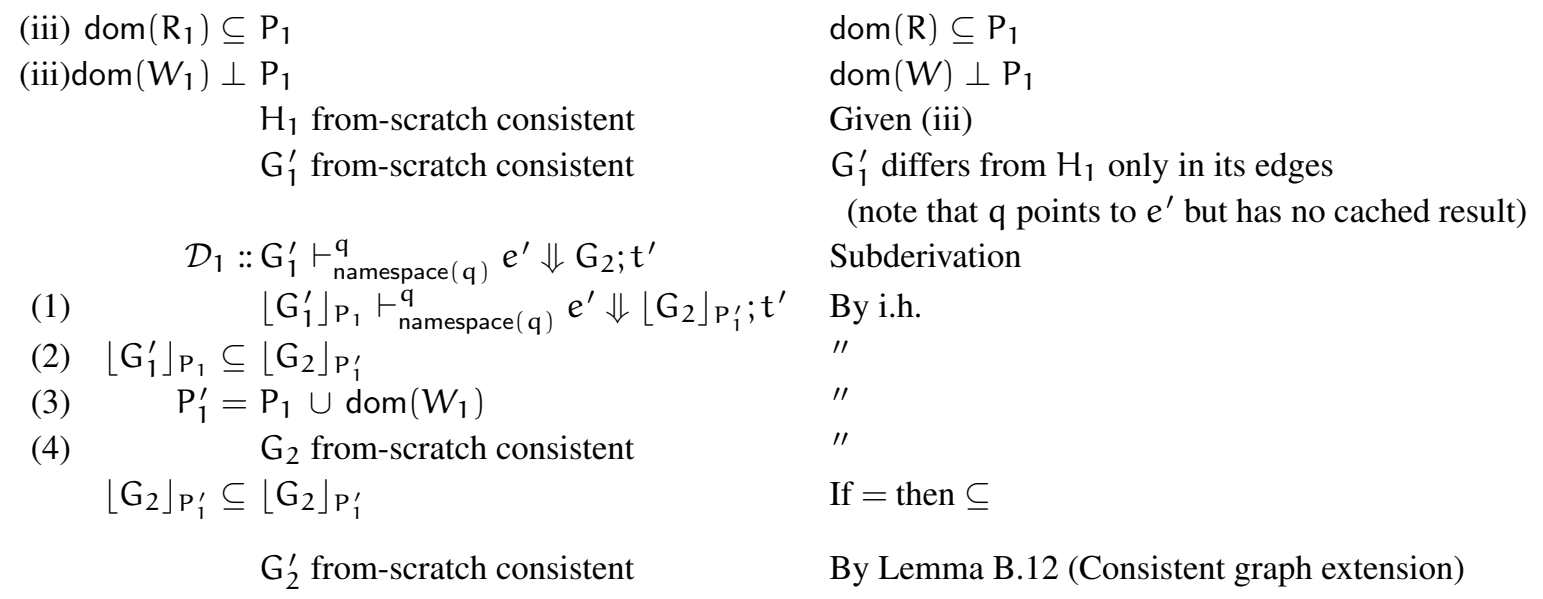

Having "stowed away" the consistency of $e^{\prime}$, we can move on to $\mathcal{D}_{2}$.

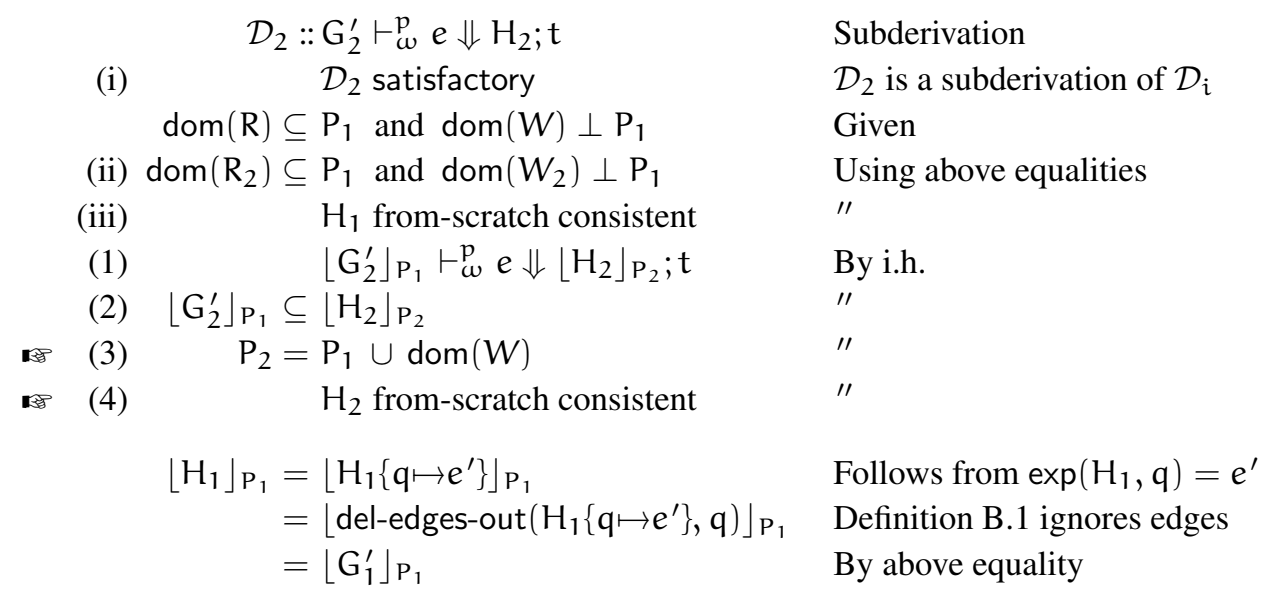

We need to show $\left\lfloor G_{1}^{\prime}\right\rfloor_{P_{1}}=\left\lfloor G_{2}\right\rfloor P_{1}$. That is, evaluating $e^{\prime}$-which will be done inside the reference derivation's version of $\mathcal{D}_{2}$-doesn't change anything in $P_{1}$.

Fortunately, we know that $\operatorname{dom}(W) \perp \mathrm{P}_{1}$ and $\operatorname{dom}\left(\mathrm{W}_{1}\right) \subseteq \operatorname{dom}(W)$. Therefore $\operatorname{dom}\left(W_{1}\right) \perp \mathrm{P}_{1}$.

By Lemma B.5 Respect for write-set), $G_{2}$ agrees with $G_{1}^{\prime}$ on $\operatorname{dom}\left(G_{1}^{\prime}\right)-\operatorname{dom}\left(W_{1}\right)$. Since $W_{1}$ is disjoint from $P_{1}$, we have that $G_{2}$ agrees with $G_{1}^{\prime}$ on $P_{1}$.

Therefore $\left\lfloor\mathrm{G}_{1}^{\prime}\right\rfloor \mathrm{P}_{1}=\left\lfloor\mathrm{G}_{2}\right\rfloor \mathrm{P}_{1}$.

Now we'll show that $\left\lfloor G_{2}\right\rfloor_{P_{1}}=\left\lfloor G_{2}^{\prime}\right\rfloor_{P_{1}}$, that is, $\left\lfloor G_{2}\right\rfloor_{P_{1}}=\left\lfloor G_{2}\left\{q \mapsto\left(e^{\prime}, t^{\prime}\right)\right\}\right\rfloor P_{1}$.

- If $\mathrm{q} \notin \mathrm{P}_{1}$ then this follows easily from Definition $\mathrm{B} .1$

- Otherwise, $q \in P_{1}$. We have $\exp \left(G_{1}^{\prime}, q\right)=e^{\prime}$ and therefore $\exp \left(G_{2}, q\right)=e^{\prime}$, so updating $G_{2}$ with $q$ pointing to $e^{\prime}$ doesn't change the restriction.

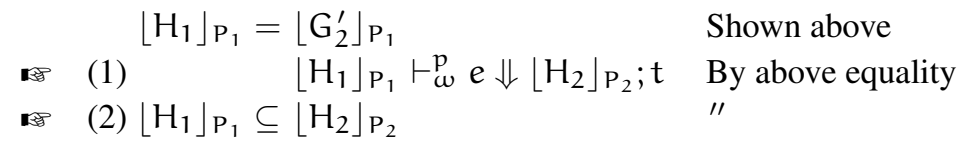

- Case $\frac{H_{1}(q)=(e, t) \quad \text { all-clean-out }\left(H_{1}, q\right)}{H_{1} \vdash_{\omega}^{p} \text { force }(\text { thk } q) \Downarrow H_{1},(p, \text { obs } t, \text { clean, } q) ; t}$ Eval-forceClean 


$$
\begin{aligned}
& \mathrm{H}_{2}=\left(\mathrm{H}_{1},(\mathrm{p}, \text { obs } \mathrm{t}, \text { clean }, \mathrm{q})\right) \quad \text { Given } \\
& \mathrm{H}_{1}(\mathrm{q})=(e, \mathrm{t}) \quad \text { Premise } \\
& \mathrm{H}_{1} \text { from-scratch consistent over } \mathrm{P}_{1} \quad \text { Given (iii) } \\
& \mathcal{D}_{\mathrm{q}} \text { satisfactory Definition B.9 (i) } \\
& \operatorname{dom}\left(\mathrm{R}_{\mathrm{q}}\right) \subseteq \mathrm{P}_{\mathrm{q}} \quad \text { " (ii) } \\
& \operatorname{dom}\left(W_{\mathrm{q}}\right) \perp \mathrm{P}_{\mathrm{q}} \\
& \mathrm{R}=\left(\mathrm{R}_{\mathrm{q}}, \mathrm{q}:(e, \mathrm{t})\right) \quad \text { By Definition B.2 for Eval-forceClean }\left(\mathcal{D}^{\prime}=\mathcal{D}_{\mathrm{q}}\right) \\
& W=W_{q}
\end{aligned}
$$

To show that $\mathcal{D}_{q}$ is consistent, we use assumption (iii) that $\mathrm{H}_{1}$ is from-scratch consistent. We have $q:(e, t)$ in $\mathrm{H}_{1}$. By Definition B.10, $\mathcal{D}_{\mathrm{q}}:: \mathrm{G}_{\mathrm{q}} \vdash_{\omega_{\mathrm{q}}}^{\mathrm{p}_{\mathrm{q}}} \mathrm{e} \Downarrow \mathrm{G}_{\mathrm{q}}^{\prime} ; \mathrm{t}$ is from-scratch consistent for some $\mathrm{P}_{\mathrm{q}} \subseteq \operatorname{dom}\left(\mathrm{G}_{\mathrm{q}}\right)$ up to $\mathrm{H}_{1}$. Now we turn to Definition B.9

$$
\begin{aligned}
& \left\lfloor\mathrm{G}_{\mathrm{q}}\right\rfloor_{\mathrm{P}_{\mathrm{q}}} \vdash_{\omega_{\mathrm{q}}}^{\mathrm{p}_{\mathrm{q}}} \in \Downarrow\left\lfloor\mathrm{G}_{\mathrm{q}}^{\prime}\right\rfloor_{\mathrm{P}_{\mathrm{q}}} \cup \operatorname{dom}\left(W_{\mathrm{q}}\right) ; \mathrm{t} \quad \text { By Definition B.9 (1) }
\end{aligned}
$$



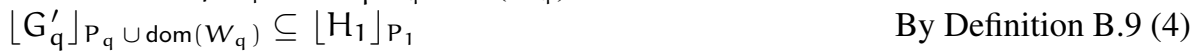

$$
\begin{aligned}
& \left\lfloor\mathrm{H}_{1}\right\rfloor_{\mathrm{P}_{1}} \vdash_{\omega_{\mathrm{q}}}^{\mathrm{p}_{\mathrm{q}}} \mathrm{e} \Downarrow\left\lfloor\mathrm{H}_{1}\right\rfloor_{\mathrm{P}_{1} \cup \operatorname{dom}\left(W_{\mathrm{q}}\right)} ; \mathrm{t} \quad \text { By Lemma B.8 Weakening (non-incremental) }
\end{aligned}
$$

Let $P_{2}$ be $P_{1} \cup \operatorname{dom}\left(W_{q}\right)$.

$$
\begin{aligned}
& \left\lfloor\mathrm{H}_{1}\right\rfloor \mathrm{P}_{1} \vdash \vdash_{\omega}^{\mathrm{p}} \mathrm{e} \Downarrow\left\lfloor\mathrm{H}_{1}\right\rfloor_{\mathrm{P}_{2}} ; t \quad \text { By above equality } \\
& \left\lfloor\mathrm{H}_{1}\right\rfloor_{\mathrm{P}_{1}} \vdash_{\omega}^{\mathrm{p}} \text { force }(\text { thk } \mathrm{q}) \Downarrow\left\lfloor\mathrm{H}_{1}\right\rfloor_{\mathrm{P}_{2}} ; \mathrm{t} \quad \text { By rule Eval-forcePlain } \\
& \left\lfloor H_{1}\right\rfloor_{\mathrm{P}_{1}} \vdash_{\omega}^{\mathrm{p}} \text { force }(\text { thk } q) \Downarrow\left\lfloor\mathrm{H}_{1},(\mathrm{p}, \text { obs } t \text {, clean, } q)\right\rfloor_{\mathrm{P}_{2}} ; t \quad \text { By Definition B.1 } \\
& \text { (1) }\left\lfloor\mathrm{H}_{1}\right\rfloor_{\mathrm{P}_{1}} \vdash_{\omega}^{\mathrm{p}} \text { force }(\text { thk } \mathrm{q}) \Downarrow\left\lfloor\mathrm{H}_{2}\right\rfloor_{\mathrm{P}_{2}} ; \mathrm{t} \quad \text { By above equality }
\end{aligned}
$$$$
\left\lfloor\mathrm{H}_{1}\right\rfloor_{\mathrm{P}_{1}} \subseteq\left\lfloor\mathrm{H}_{1}\right\rfloor_{\mathrm{P}_{2}} \quad \text { By a property of Definition B.1 }
$$$$
\text { (2) }\left\lfloor\mathrm{H}_{1}\right\rfloor \mathrm{P}_{1} \subseteq\left\lfloor\mathrm{H}_{2}\right\rfloor \mathrm{P}_{2} \quad \text { By a property of Definition } \overline{B .1}
$$$$
\text { (3) } \quad P_{2}=P_{1} \cup \operatorname{dom}(W) \quad W=W_{q}
$$

(4) $\mathrm{H}_{2}$ from-scratch consistent $\mathrm{H}_{2}$ differs from $\mathrm{H}_{1}$ only in its edges

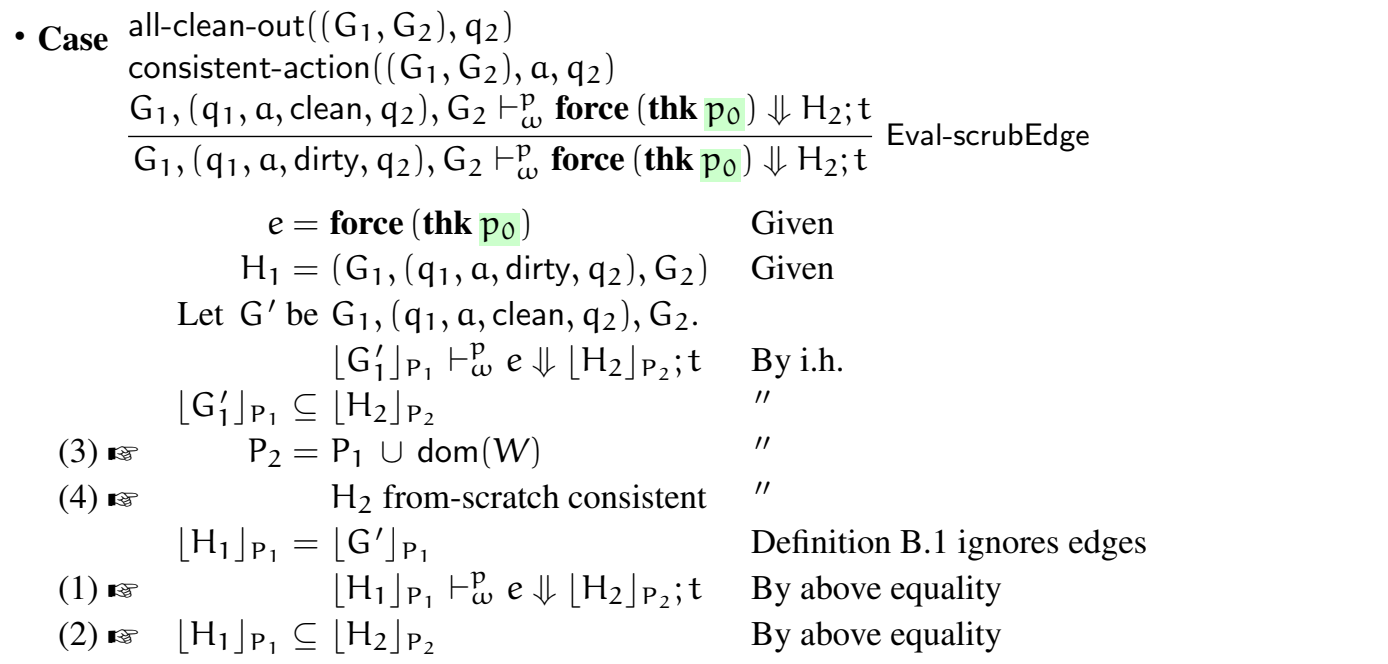

